\title{
COMMENTO DELLE SCELTE DELLE FORME VERBALI NEI CLOZE DEGLI STUDENTI DI ITALIANISTICA E DI TRADUZIONE (IDENTIFICAZIONE DEI PUNTI CRITICI E CONFRONTO TRA I GRUPPI)
}

\section{INTRODUZIONE}

Il presente contributo ha come argomento le scelte delle forme verbali italiane nei cloze compiute dagli studenti universitari di italiano, e il variare di questa prestazione linguistica in dipendenza dagli anni di studio e dal corso di laurea frequentato: il corso di Lingua e letteratura italiana da un lato e i corsi di Traduzione e di Mediazione interlinguistica dall'altro.

Dopo un breve confronto tra le caratteristiche dei sistemi verbali italiano e sloveno, condotto allo scopo di evidenziare le difficoltà di un apprendente slovenofono, saranno presentati il test e il questionario. Sarà discusso il cloze come metodo con cui non soltanto si mette il soggetto testato di fronte al compito di riempire il testo con le forme verbali che ritiene appropriate, ma ancora prima gli si chiede implicitamente di interpretare felicemente il messaggio del testo lacunoso. Si vedrà, in seguito, che gli errori e le imprecisioni degli studenti non sono dovuti tanto a problemi di comprensione del mondo testuale quanto piuttosto a difficoltà relative alla scelta della forma verbale appropriata. Passerò quindi in rassegna i dati delle analisi, soffermandomi sulla presentazione dei punti critici, sul commento delle scelte e sul confronto tra i gruppi. In base ai risultati ottenuti, cercherò di offrire, infine, dei suggerimenti di carattere didattico.

\section{PARTE TEORICA}

Secondo Miklič (1997: 479) la scelta delle forme verbali dipende da tutta una serie di parametri: da quelli che riguardano il contenuto del messaggio e il modo in cui esso viene valutato - temporalità assoluta, temporalità relativa, ${ }^{1}$ tipo di azione (Aktionsart), modalità ecc. - e da quelli che riguardano le caratteristiche formali e i modi di presentazione - tipo di testo, varietà di lingua, adozione o presa di distanza, orientamenti dello

\footnotetext{
* Indirizzo dell'autrice: Filozofska fakulteta, Oddelek za romanske jezike in književnosti, Aškerčeva 2, 1000 Ljubljana, Slovenia. Email: mirjampremrl@gmail.com

${ }^{1}$ Con «temporalità assoluta» si intende la posizione temporale dell'azione rispetto al momento dell'enunciazione: passato, avvenire, attualità, attualità allargata, extratemporalità. Con «temporalità relativa», invece, si indica la relazione temporale tra l'azione in questione e un altro punto di riferimento, diverso dal momento dell'enunciazione: anteriorità, simultaneità, posteriorità, contiguità e indipendenza temporale (assenza di tale relazione).
} 
sguardo, ${ }^{2}$ messa in rilievo e aspettualità, ${ }^{3}$ meccanismi sintattici, ${ }^{4}$ procedimenti narrativi, ${ }^{5}$ modi di inserimento di altri discorsi ${ }^{6}$ ecc. La realtà sottostante ai parametri menzionati viene codificata in modo diverso nelle diverse lingue, il che può comportare delle difficoltà nel processo di apprendimento e di acquisizione di una lingua straniera.

Già da un superficiale confronto tra lo sloveno e l'italiano si vede che l'italiano dispone di una vasta gamma di paradigmi verbali finiti e infiniti, mentre lo sloveno ne vanta pochi soltanto; tra essi, quelli infiniti sono caratterizzati da un uso molto ristretto (cfr. Miklič 1992a: 197).

In italiano le forme verbali finite sono di norma distribuite nella maniera seguente: indicativo (presente $(\mathrm{Pr})$, passato prossimo $(\mathrm{PP})$, futuro semplice $(\mathrm{F})$, futuro composto $(\mathrm{FF})$, imperfetto (IM), passato remoto (PR), trapassato prossimo (TP), trapassato remoto (TR)), congiuntivo (presente (Pr'), passato (PP'), imperfetto (IM'), trapassato (TP')), condizionale (condizionale semplice (C), condizionale composto (CC)), imperativo (imperativo (ImP)). Si classificano tra le forme verbali infinite le seguenti forme: infinito semplice (INF), infinito composto (INFF), gerundio semplice $(\mathrm{G})$, gerundio composto (GG), participio (PART), participio presente (PARTPr). Per quel che riguarda, invece, le forme verbali finite in sloveno appartengono all'indicativo il sedanjik (il «presente» sloveno, SED), il prihodnjik (il «futuro» sloveno, PRIH), il preteklik (il «preterito» sloveno, PRET) e il predpreteklik (il «trapassato» sloveno); al condizionale il pogojnik (il «condizionale» sloveno, POG) e il pretekli pogojnik (il «condizionale passato» sloveno); all'imperativo il velelnik (cfr. Miklič/Ožbot 2007: 66-67; Toporišič 2000: 510-511). Il predpreteklik e il pretekli pogojnik si adoperano molto raramente (Miklič 1992a: 197).

Questo breve confronto delle due realtà fa già intuire l'esistenza di differenze profonde tra le due lingue; la vera diversità consiste, tuttavia, non solo nella quantità delle forme verbali, ma soprattutto nel loro impiego. Le due lingue differiscono sensibilmente nell'espressione della temporalità relativa, dell'aspettualità, della modalità e della sintassi (cfr. Miklič/Ožbot 2007: 69-70). L'italiano offre al parlante una serie di paradigmi verbali atti ad indicare contemporaneamente l'ubicazione dell'azione tanto

\footnotetext{
${ }^{2}$ Con questo termine ci si riferisce a tecniche espositive-narrative quali ordine naturale, flash-back, flash-forward e preludio. Si ha l'ordine naturale quando l'ordine dei predicati del testo coincide con l'ordine cronologico delle azioni nella realtà extralinguistica ovvero nel mondo testuale. Si parla invece del flash-forward quando in un punto della narrazione il narratore riporta uno o più eventi posteriori ma realmente accaduti, per poi tornare ai fatti della storia principale ossia alle azioni nel fuoco narrativo. Per una presentazione più dettagliata del flash-back e del preludio si vedano 2.8.1 e 2.8.5.

${ }^{3}$ Con le due espressioni ci si riferisce alla presentazione delle azioni in primo piano per mezzo di un paradigma perfettivo, o in secondo piano per mezzo di un paradigma imperfettivo.

${ }^{4}$ Con l'espressione «meccanismi sintattici» si pensa all'uso delle forme in vari tipi di strutture sintattiche.

${ }^{5}$ Con l'espressione «procedimenti narrativi» si pensa alle tecniche espositive relative alla scelta degli insiemi di forme verbali per raccontare il passato, cioè ai procedimenti fondamentale, storico, combinato e misto. Nei due più frequenti, fondamentale e storico, le azioni centrali vengono di norma segnalate nel primo caso tramite il passato remoto (o passato prossimo), nel secondo caso tramite il presente storico. Nei cloze del presente contributo si ha il procedimento narrativo fondamentale.

${ }^{6} \mathrm{Ne}$ fanno parte ad. es. il discorso diretto (DD), il discorso indiretto (DI) e il discorso indiretto libero (DIL).
} 
rispetto al momento dell'enunciazione quanto rispetto ad un altro punto di riferimento e quindi dispone di paradigmi distinti per l'espressione della cronologia relativa nel non-passato (PP/PP', $\mathrm{Pr} / \mathrm{Pr}$ ', $\mathrm{F} / \mathrm{Pr} / \mathrm{Pr}$ ') rispetto al passato (TP/TP', IM/IM', $\mathrm{CC} / \mathrm{IM} / \mathrm{IM}$ '). Questi paradigmi verbali si usano per segnalare un determinato rapporto di tipo relativo nei costrutti sintattici più frequenti (giustapposizione (frasi indipendenti), periodi con dipendenti relative, causali, completive e alcuni altri). In questi costrutti la situazione in sloveno è simile a quella italiana nel non-passato, dove con il PRET, il SED e il PRIH ci si riferisce rispettivamente alle azioni anteriori, simultanee e posteriori. Diverso è l'uso delle forme verbali per la sfera del passato. Mentre nelle completive i rapporti di tipo relativo vengono segnalati - anche stavolta per mezzo del PRET, del SED e del PRIH, come nel non-passato -, nei costrutti indipendenti, relativi e causali si fa uso del PRET, lasciando al destinatario il compito di interpretare la cronologia relativa per mezzo degli elementi di co- e contesto (Miklič 1992a: 195-201).

Un altro punto importante riguarda le differenze nel funzionamento del sistema aspettuale sloveno rispetto a quello italiano. ${ }^{7}$ In sloveno, in linea di massima, il tipo di azione in buona parte incide sulla scelta di uno dei due elementi in opposizione, giacché con l'impiego del nedovršnik (ND) (il «verbo imperfettivo» sloveno) viene messa in rilievo la fase durativa delle azioni omogenee (ateliche) nonché di quelle teliche che dispongono di una tale fase, mentre con il dovršnik (D) (il «verbo perfettivo» sloveno) viene indirizzata l'attenzione verso la fase puntuale dell'azione telica, ma (con i verbi fasali) anche verso la fase iniziale ${ }^{8}$ o quella finale di quella atelica (Miklič 2007: 92, 9596, 99). ${ }^{9}$ Sul versante italiano, invece, quando l'azione omogenea o telica, di carattere singolo o iterativo, viene considerata nella sua globalità, si fa ricorso al perfetto, mentre l'espressione della visione parziale di un'azione qualsiasi, ${ }^{10}$ osservata da un punto passato rispetto al quale l'azione è simultanea, si affida all'imperfetto (Miklič 2007:

\footnotetext{
${ }^{7}$ Per distinguere le due realtà Miklič (1981: 8) propone l'impiego del termine vid in riferimento alla situazione in sloveno e del termine ottica per la situazione in italiano.

${ }^{8}$ Qualche volta la fase iniziale può essere esplicitata con un verbo incoativo apposito.

${ }^{9}$ Secondo la classificazione vendleriana rientrano tra le azioni ateliche gli stativi (state: essere malato) e i processi (activity: piangere), mentre fanno parte delle azioni teliche le realizzazioni (accomplishment: scrivere una lettera) e i conseguimenti (achievement: restituire) (Bertinetto 2003: 14). Sebbene l'azione atelica disponga anche di un inizio e di una fine, cioè di due fasi puntuali, la sua fase principale - quella che di norma viene messa in rilievo - è la fase durativa, per cui tale azione viene presentata per mezzo del ND (jokati [piangere]). Le fasi iniziale e finale, che si realizzano per mezzo del D, potrebbero essere classificate come situazioni di tipo conseguimento (zajokati [mettersi a piangere/cominciare a piangere], začeti/nehati delati, končati z delom [cominciare a/smettere di, finire di lavorare]). Le azioni di tipo conseguimento sono caratterizzate dalla sola fase puntuale (počiti [esplodere]), ma in alcuni casi possono disporre sia di una fase preparatoria durativa che di una fase puntuale (prihajati (ND) na vrh/priti (D) na vrh [stare per raggiungere la vetta/raggiungere la vetta]. Dato che in questo tipo di azioni viene, di norma, messa in rilievo la fase puntuale i conseguimenti sono perlopiù esplicitati per mezzo del D. Le realizzazioni, invece, sono caratterizzate sia dalla fase preparatoria durativa che dalla fase puntuale conclusiva. Quando si vuole presentare tutta l'azione, ponendo l'accento sul punto finale, viene usato il D. Quando, invece, si presenta tutta l'azione, ma senza dirigere l'attenzione sul punto finale, o quando l'attenzione si dirige verso la fase durativa, si ricorre all'impiego del ND (Miklič 2007: 92, 95-96).
}

10 Sono da escludere azioni momentanee puntuali, se non nel caso dell'impiego dell'imperfetto stilistico (cfr. Miklič 1981: 73). 
93). ${ }^{11}$ Mentre in italiano la distinzione tra l'aspetto perfettivo e imperfettivo, sul lato espressivo, concerne soltanto alcuni paradigmi verbali (il PR/PP/TP in opposizione all'IM) in sloveno ogni forma verbale si manifesta o nella variante perfettiva o in quella imperfettiva, oppure - più raramente in quella biaspettuale..$^{12}$

Da quanto emerge da alcuni studi - e in particolare da quelli incentrati sull'impiego dei paradigmi verbali italiani in dipendenza dai costrutti sintattici da parte degli studenti universitari e ginnasiali - le difficoltà in questo campo non sono del tutto superate nemmeno nei casi in cui l'italiano è una lingua dell'ambiente sociale, oppure vanta una forte presenza soprattutto a causa dei media: non lo sono nemmeno a livello universitario (Mertelj 2008: 349). A questo punto si potrebbe estendere il concetto all'impiego delle forme verbali in generale e ipotizzare che l'uso corretto dei paradigmi verbali sia conseguenza dell'apprendimento cosciente piuttosto che dell'acquisizione spontanea. ${ }^{13} \mathrm{In}$ quest'ottica persino gli anni di contatto con la lingua e le preconoscenze non sarebbero in grado di eliminare del tutto le difficoltà nella scelta dei paradigmi del sistema verbale italiano. L'asserzione sembra trarre conferma da altri due studi di Miklič (1992b: 475 e 1998a: 373); il primo si incentra appunto sull'analisi degli aspetti problematici dell'apprendimento dell'italiano da parte di un madrelingua sloveno. Di fronte alle difficoltà individuate l'autrice chiama di nuovo in causa la codificazione diversa della temporalità e dell'aspettualità in sloveno, ossia l'interferenza della madrelingua (Miklič 1992b: 475). La problematica dell'interferenza è stata affrontata anche da Sicherl (1998: 356), che, in base all'analisi dell'uso delle preposizioni negli studenti d'inglese, sostiene che in condizioni di mancato sapere metalinguistico o di altre difficoltà nell'usare una lingua straniera si ricorre alla madrelingua. La stessa realtà è stata osservata di recente da Ožbot (2009: 26), secondo la quale gli studenti componendo dei testi in una lingua straniera in qualche modo «traducono» continuamente dalla propria o da un'altra lingua che sentono vicina.

Influenzati dallo sloveno gli studenti d'italiano dovrebbero incontrare difficoltà sebbene in misura piuttosto limitata grazie all'insegnamento esplicito - nell'espressione dell'anteriorità nel passato nell'ambito delle frasi relative e indipendenti (i casi di flash-back). Potrebbe risultare difficoltosa l'espressione della temporalità relativa tra le azioni passate nel caso dei discorsi inseriti, indiretto nell'ambito delle completive e indiretto libero nell'ambito delle indipendenti. Si dovrebbe notare la tendenza, negli

11 Le azioni colte globalmente vengono denominate da Miklič (1981: 76) «azioni di primo piano», mentre le azioni viste corsivamente sono definite «azioni di secondo piano». L'atteggiamento verso una rappresentazione di azioni globale è chiamato «narrazione», a differenza della tendenza a presentare azioni in modo corsivo, designata con il termine «descrizione» (Miklič 2007: 93). Con il termine «visione corsiva» ci si riferisce alla visione dall'interno dell'azione. L'espressione è stata presa da uno studio di Hermann («Objektive und subjektive Aktionsart», 1927) (Miklič 2007: 86).

12 In alcuni casi la stessa forma può comparire come dovršnik e nedovršnik.

13 Gli effetti positivi del sapere acquisito sull'uso dei paradigmi verbali comunque non si possono del tutto escludere. La problematica è sfiorata da Mertelj (2008: 362) nella sua presentazione sintetica dell'uso dei paradigmi verbali in alcuni costrutti sintattici da parte degli studenti ginnasiali. Se non altro la familiarità con la lingua incide sicuramente su un livello più alto di comprensione testuale e, di conseguenza, su una maggiore disinvoltura, il che può condurre a risultati migliori. Senonché in certi studenti appunto questo pregio pare essere causa di un mancato interesse nei confronti dell'apprendimento dei contenuti grammaticali. 
studenti, ad identificare l'uso del perfetto con l'uso del dovršnik e l'uso dell'imperfetto con l'uso del nedovršnik. Anche l'impiego attivo del preludio, praticamente sconosciuto nello sloveno odierno (Miklič 1998b: 187), dovrebbe rivelarsi tutt'altro che facile. Infine non sono da escludere difficoltà nell'uso del congiuntivo.

\section{PARTE EMPIRICA}

\subsection{Preparazione e fini del test}

Per poter analizzare le prestazioni degli studenti nell'uso delle forme verbali - soprattutto nei punti ritenuti critici - nonché il loro evolversi in dipendenza dagli anni e dall'indirizzo di studio - ho elaborato un test composto da due parti. La prima consisteva di cinque testi tratti da due numeri della rivista La settimana enigmistica (2008), la seconda, invece, di un brano tratto dal romanzo Il giardino dei Finzi Contini (GFC). Tutti e sei sono stati trasformati in altrettanti cloze con, tra parentesi, le forme verbali all'infinito.

Ho scelto il cloze come tipo di esercizio perché nell'insegnamento moderno delle lingue straniere esso viene spesso usato anche per verificare le capacità dei discenti in relazione agli usi delle forme verbali. Rispetto a qualche altro esercizio linguistico - ad es. alla traduzione - il cloze, che indirizza l'attenzione del discente solo verso alcuni punti del testo, mentre tutto il resto è già dato, sembrerebbe a prima vista un compito più facile. $\mathrm{Ma}$ in realtà il soggetto testato si trova davanti a un duplice compito. Sulla base del testo lacunoso deve in primo luogo capire il contenuto espresso dall'autore, cioè ricostruire felicemente il mondo testuale; soltanto in seguito potrà completare il cloze con le forme verbali che ritiene appropriate, sia in base alle regole grammaticali apprese sia in modo automatico.

La settimana enigmistica (LSE) è stata scelta per offrire ai partecipanti testi completi e brevi di carattere divulgativo, e, quindi, non troppo complicati dal punto di vista lessicale, strutturale o contenutistico. I testi, di tipo prevalentemente narrativo, erano inoltre caratterizzati da una grande varietà di rapporti temporali e di conseguenza da un uso svariato dei paradigmi verbali.

Ecco i testi trasformati nei cloze. Il lettore è invitato a completarli e a confrontare in seguito la propria scelta con la presentazione dei fenomeni e con le soluzioni. Ad ogni soluzione italiana ho aggiunto - per evidenziare le differenze nel funzionamento dei sistemi verbali italiano e sloveno - anche la forma verbale con cui potrebbe essere resa la stessa realtà nel testo sloveno.

\section{A. SALVATI DAL CANE \\ Jean-Daniel e i suoi due bambini A.1 (partire) per una gita sulla neve nei dintorni di Villars-sur- Ollon (Svizzera) a bordo della slitta A.2 (trainare) dal loro cane Shady, quando all'improvviso una slavina li aveva travolti e sepolti. I soccorritori li hanno raggiunti solo dopo un certo tempo,

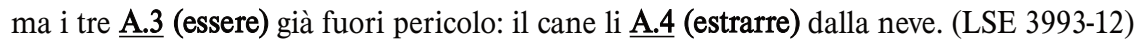

A.1: L'osservazione attenta del cotesto (aveva travolti e sepolti) suggerisce l'uso del trapassato prossimo dell'indicativo (erano partiti (TP)/so se odpravili (PRET-D)/). I primi avvenimenti (era partito, aveva travolti e sepolti) costituiscono una specie di preambolo rispetto agli avvenimenti focali (li hanno raggiunti). Si tratta del cosiddetto preludio. A.2: La struttura e il conte- 
nuto richiedono l'impiego della forma verbale infinita PART (trainata/trainati /je vlekel (PRET-ND)/). A.3: L'azione è simultanea rispetto all'azione passata - (li) hanno raggiunti (erano (IM) /so bili (PRET-ND)/). A.4: L'azione è anteriore rispetto a (li) hanno raggiunti e erano fuori pericolo (aveva estratti (TP) /je izkopal (PRET-D)/). Si tratta di un flash-back (anteriorità nell'ambito della indipendente).

\section{B. MARITO ABBANDONATO}

Tempo fa, il signor Norman Long di Saint Petersburg, una città della Florida, intentò una causa di divorzio contro la moglie Lizzie, accusandola di abbandono del tetto coniugale. La donna B.1 (allontanarsi) da casa poco dopo le nozze, celebrate più di quarant'anni prima, e non vi B.2 (fare più) ritorno. Al giudice che gli $\underline{B} .3$ (chiedere) come mai $\underline{\mathbf{B} .4}$ (aspettare) tanto a denunciare la scomparsa, il marito abbandonato rispose: «Ho sempre sperato che prima o poi B.5 (tornare) da me...» (LSE 3993-12)

B.1/B.2: Le azioni (verbalizzate nella indipendente) sono anteriori rispetto alle azioni passate nel fuoco narrativo - intentò, chiese, rispose. Si tratta di un flash-back (si era allontanata (TP) /je odšla (PRET-D)/; non aveva più fatto (TP)/se ni več vrnila (PRET-D)/). B.3: L'azione è contigua all'azione rispose (chiese (PR) /je vprašal (PRET-D)/). L'azione potrebbe essere interpretata anche come anteriore rispetto a rispose (aveva chiesto (TP) /je vprašal (PRET-D)/). B.4: L'azione è verbalizzata nell'ambito della completiva (il discorso indiretto del giudice) ed è anteriore rispetto all'azione chiese della frase citante. Nelle dipendenti interrogative indirette introdotte da chiedere si possono usare sia il congiuntivo che l'indicativo (Wandruszka 2001: 471) (avesse aspettato/aveva aspettato (TP'/TP) /je čakal (PRET-ND)/). B.5: L'azione, verbalizzata nell'ambito della completiva, è posteriore rispetto all'azione passata ho sperato (sarebbe tornata (CC)/se bo vrnila (PRIH-D)/). Per sottolineare la sfumatura modale può essere usato l'IM' (tornasse).

\section{GIULIO II}

Verso la fine del 1510, papa Giulio II C.1 (incominciare) a sfoggiare una folta barba, che

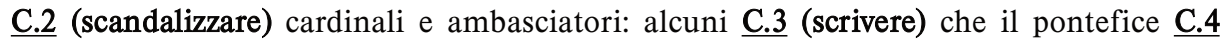
(somigliare) a un orso, altri lo C.5 (paragonare) invece a un eremita. In realtà, con tale

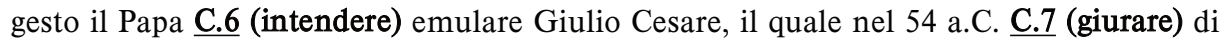
non radersi finché $\underline{\mathbf{C} .8}$ (non vendicare) la morte dei propri uomini $\underline{\mathbf{C}} \mathbf{9}$ (massacrare) dai Galli. Secondo quanto riportato da un cronista bolognese, Giulio II $\underline{\text { C.10 }}$ (essere determi-

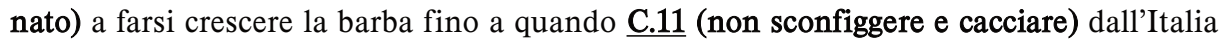
il re francese Luigi XII. (LSE 3993-4)

Quando in un testo, caratterizzato da un registro piuttosto elevato, l'argomento è di tipo storico il paradigma più spesso usato per denotare azioni centrali è il passato remoto (cfr. Bertinetto/Squartini 1996: 389-391, 400, 413-414). C.1: Si tratta di un'azione centrale nel passato (incominciò (PR) /je začel (PRET-D)/). C.2: L'azione è presentata come simultanea all'azione passata sfoggiare (scandalizzava (IM) / nad katero so se zgražali (PRET-ND)/). Potrebbe essere presentata anche come contigua (scandalizzò (PR)). C.3-C.5: In italiano c'è la tendenza a presentare le azioni nell'ambito della coordinazione copulativa e dell'asindeto in una stessa ottica (Miklič 1981: 82-83) (scrissero-paragonarono (PR-PR); scrivevano-paragonavano (IM-IM) /so pisali/so zapisali-so primerjali (PRET-ND/D, PRET-ND)/). C.4: 
L'azione, verbalizzata nella completiva (discorso indiretto) è simultanea all'azione scrissero (somigliava (IM) /spominja (SED-ND)/). C.6: L'azione è simultanea rispetto a incominciò a sfoggiare (intendeva (IM) /je nameraval (PRET-ND)/). C.7: L'azione è anteriore rispetto alle azioni incominciò a sfoggiare e intendeva (aveva giurato (TP) /je prisegel (PRET-D)/). C.8: L'azione è anteriore rispetto a di non radersi che è sua volta posteriore rispetto a aveva giurato. L'azione è verbalizzata in una temporale nell'ambito della completiva (finché non avesse vendicato (TP') /dokler ne bo maščeval (PRIH-D/ND)/). C.9: La struttura sintattica e il contenuto richiedono l'impiego della forma verbale infinita PART (massacrati /so jih pomorili (PRET-D)/). C.10: L'azione è simultanea a incominciò a sfoggiare (era determinato (IM) /je bil odločen (PRET-ND)/). C.11: L'azione è anteriore rispetto a farsi crescere che è sua volta posteriore rispetto a era determinato a. L'azione è verbalizzata in una temporale nell'ambito della completiva (fino a quando non avesse sconfitto e cacciato (TP') /dokler ne bo premagal in pregnal (PRIH-D)/).

\section{BENIAMINO}

Il biblico patriarca Giacobbe decise che al suo ultimo figlio D.1 (dare) il nome augurale di Ben Yamin (Beniamino), che in ebraico D.2 (significare) «figlio della destra», e dunque

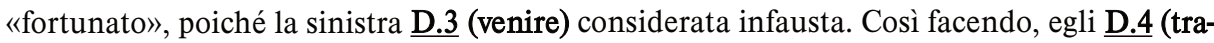
sgredire) le ultime volontà di sua moglie Rachele, che, prima di morire nel dare alla luce quel figlio, D.5 (chiedere) che il bambino D.6 (chiamare) Ben Oni, ossia «figlio del mio dolore». (LSE 3993-4)

D.1: L'azione, verbalizzata nella completiva, è posteriore rispetto all'azione passata decise (avrebbe dato (CC) /bo dal (PRIH-D)/). Anche se il verbo decidere fa parte dei verbi volitivi a cui nella dipendente segue di norma un verbo al congiuntivo (Wandruszka 2001: 420-421) l'impiego dell'IM' (desse) in D.1 non è possibile dato che il soggetto della completiva e della sovraordinata è lo stesso. L'espressione della volizione, infatti, risente della finalità e quindi non può essere autoreferenziale ( ${ }^{*}$ decise che desse). È invece possibile l'impiego della forma passiva dell'IM' (fosse dato (IM') / naj mu bo (PRIH-ND)/) appunto per la diversità dei soggetti. ${ }^{14}$ D.2: L'azione è presentata come extratemporale (significa (Pr) /pomeni (SED-ND)/). D.3: L'azione è simultanea all'azione decise (veniva considerata (IM) /so jo imeli za (PRET-ND)/). D4: Si tratta di un'azione centrale nel passato (trasgredì (PR) /ni izpolnil (PRET-D)/). D.5: L'azione è anteriore rispetto all'azione trasgredì (aveva chiesto (TP) /je prosila (PRET-ND)/). D.6: Dato che con il verbo chiedere è espressa una volizione che concerne un'azione posteriore nel passato, nella dipendente è richiesto l'uso dell'IM' (venisse chiamato/fosse chiamato (IM') / naj ga poimenujejo (naj + SED-D)/) (Wandruszka 2001: 420-421).

\section{E. I DANAI}

Secondo quanto afferma l'ingegner Felice Vinci nel libro Omero nel Baltico, le gesta dei Danai, narrate nei poemi omerici, E.1 (non svolgersi) nel Mediterraneo, ma nei mari del Nordeuropa. Partendo da uno scritto di Plutarco, nel quale lo storico greco E.2 (sostenere) che l'isola di

\footnotetext{
14 Ho scoperto questa tendenza esaminando i casi nel corpus La Repubblica (http://dev.sslmit.unibo.it/corpora/query.php?mode=simple\&path=\&name=Repubblica).
} 
Calipso, Ogigia, è situata a cinque giorni di navigazione dalla Britannia, Vinci E.3 (analizzare) numerosi toponimi dell'Europa settentrionale, sorprendentemente simili a quelli omerici, nonché svariati fenomeni naturali, come gli enormi gorghi d'acqua detti maelstrom, che E.4 (ricordare) Scilla e Cariddi. Egli è quindi giunto a sostenere che i Danai (nome con cui Omero

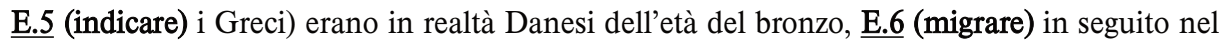
Mediterraneo, dove E.7 (attribuire) alle nuove terre i toponimi dei luoghi natii. (LSE 3976-12)

E.1: Si tratta dell'azione passata riguardo alla quale il produttore del testo esprime delle riserve (non si sarebbero svolte (CC) /naj bi se ne odvijala/se baje niso odvijala (naj + POG-ND/baje ${ }^{15}+$ PRETND)/). E.2: Dall'osservazione attenta del cotesto con il Pr (è situata) nella dipendente completiva (che esclude la possibilità dell'impiego dell'IM ( ${ }^{*}$ sosteneva) nella sovraordinata dato che la simultaneità nel passato non può essere resa per mezzo del Pr nella dipendente) si può dedurre la posizione extratemporale dell'azione sostenere e quindi la necessità di renderla per mezzo del Pr (sostiene /trdi (SED-ND)/). E.3: L'azione è anteriore rispetto all'azione afferma (ha analizzato (PP) /je analiziral (PRET-D/ND)/). E.4: Si tratta di un'azione extratemporale (ricordano (Pr)/spominjajo (SED-ND)/). E.5: Con l'impiego dell'IM (indicava /je označeval (PRET-ND)/) l'opinione di Omero, vista corsivamente (a proposito del termine «corsivo» si veda la nota 11), è legata al passato. In questo modo si contrappone in senso non solo semantico ma anche temporale alle tesi di Vinci (è giunto a sostenere) che invece sono legate all'attualità. Per questo motivo la scelta dell'IM è migliore rispetto ad un'altra scelta possibile, il Pr (indica /označuje (SED-ND)/) che sarebbe usato per presentare l'azione come extratemporale. E.6: La struttura e il contenuto richiedono l'impiego della forma verbale infinita PART (migrati /so se preselili (PRET-D)/). E.7: Azione centrale nel passato senza legami con il momento attuale (attribuirono (PR) /so poimenovali (PRET-D)/).

Il brano tratto dal romanzo, essendo un testo letterario, è caratterizzato da una complessità maggiore. Inoltre, è anche più lungo e meno completo dal punto di vista contenutistico. Per rimediare a quest'ultimo difetto il cloze è stato preceduto da una breve introduzione con cui si cercava di dare informazioni essenziali sul contenuto del romanzo nonché sui personaggi e sullo svolgimento della trama fino al momento dell'incontro - presentato nel brano - dei due protagonisti Giorgio (l'io narrato) e Micòl nella camera da letto di quest'ultima.

\section{GFC}

Mi bastò entrare nella sua stanza, invece, (dopo GFC.1 (introdurmi), Perotti aveva richiuso discretamente la porta dietro le mie spalle), per vedere che mi GFC.2 (sorridere - lei) benigna, gentile, amica. Ancor più dell'esplicito invito a venire avanti, GFC.3 (essere) quel suo sorriso luminoso, pieno di tenerezza e di perdono, che mi persuase a staccarmi dal fondo buio della stanza e ad avvicinarmi.

Mi accostai dunque al letto, dalla parte dei piedi, restando lí, con le mani appoggiate alla ringhiera. Benché infilata sotto le coperte, Micòl ne rimaneva fuori con tutto il busto. GFC.4 (indossare) un pullover verde-scuro, accollato e dalle maniche lunghe, la medaglietta d'oro dello sciaddài scintillante sopra la lana della maglia, e due guanciali a sostegno della schiena.

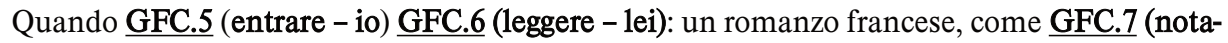

\footnotetext{
${ }^{15}$ La sfumatura della notizia riferita può essere data da un altro elemento di con- e cotesto non verbali.
} 
re - io), riconoscendo di lontano il tipo della copertina bianca e rossa; ed GFC.8 (essere) la lettura, probabilmente, piú che il raffreddore, a metterle sotto gli occhi un segno di stanchezza. No, GFC.9 (essere) sempre bella - mi GFC.10 (dire - io) - contemplandola -, forse non GFC.11 (essere mai - lei) cosí bella e attraente. (GFC 1968: 212)

GFC.1: L'azione è anteriore rispetto all'azione passata aveva richiuso. La dipendente temporale implicita richiede l'impiego della forma verbale infinita INFF (avermi introdotto /(me) je napovedal (PRET-D)/). GFC.2: L'azione è simultanea all'azione passata posteriore (per) vedere (sorrideva (IM) /se smehlja (SED-ND)/). GFC.3: Si tratta di un'azione centrale passata (visione globale). La combinazione $f u$-(che mi) persuase presenta la stessa azione resa con due predicati per dare, nell'ambito della frase scissa, enfasi al soggetto (sorriso) ( $f u$ (PR) /je bil (PRET-ND)/). GFC.4: L'azione è simultanea all'azione passata accostai (indossava (IM) / bila je (v) (PRET-ND)/). GFC.5: Con l'azione, verbalizzata in un costrutto sintatticamente indipendente dai costrutti in cui sono verbalizzate azioni nel fuoco narrativo (accostai, rimaneva, indossava), viene recuperata un'informazione anteriore flash-back (ero entrato (TP) /sem vstopil (PRET-D/). GFC.6: L'azione è simultanea all'azione anteriore nel passato ero entrato (stava leggendo/leggeva (IM) /je brala (PRET-ND)/). GFC.7: Il PR viene usato per designare un'azione di importanza secondaria - in questo caso un commento del narratore - nel flash-back caratterizzato altrimenti dall'impiego del TP (casi simili presentati in Miklič 2004: 156) (notai (PR)/sem opazil (PRET-D)/). GFC.8: L'azione è colta globalmente, però fuori del fuoco narrativo (flash-back). Si tratta di una stessa azione resa con due predicati (era stata-a metterle) nell'ambito della frase scissa (era stata (TP) /je bila (PRET-ND)/). GFC.9-11: Si tratta di azioni verbalizzate nell'ambito del discorso indiretto libero (DIL), rispettivamente simultanea e anteriore all'azione passata della frase citante dicevo (era-era stata (IM-TP) /je-je bila (SED-ND-PRET-ND)/). GFC.10: Nel romanzo abbastanza spesso si può notare la tendenza a presentare l'azione della frase citante intercalata al DIL corsivamente (v. sopra) ((mi) dicevo (IM)/sem si rekel (PRET-D)/).

Visto che la ricreazione felice del mondo testuale è essenziale per la scelta appropriata delle forme verbali - per farlo bisogna considerare il contenuto del testo come un'unità esaminando la posizione temporale di tutte le azioni e non procedere frase per frase scegliendo la forma sulla base degli elementi di cotesto vicino - ho voluto identificare le possibili difficoltà degli studenti in questo campo; perciò ho proposto ai candidati di: 1. sottolineare le espressioni sconosciute; 2. leggere la traduzione completa del testo $\mathrm{C}$ inserendo solo in seguito le forme verbali nel cloze; 3. riempire gli spazi vuoti dei cloze E e GFC, seguiti dalla traduzione slovena del testo integrale, con l'incarico di ripetere 1'esercizio (completare gli stessi cloze una seconda volta: 2E, 2GFC); 4. completare il cloze $\mathrm{D}$, tradurre il contenuto in sloveno per acquisire consapevolezza del mondo testuale e in seguito ripetere l'esercizio (completare il cloze una seconda volta: 2D); 5. rispondere alle domande riguardanti l'approccio usato nell'attività di scelta delle forme verbali. Per capire le motivazioni che li hanno guidati nella ricerca delle soluzioni ho chiesto loro di argomentare alcune scelte. I cloze saranno considerati in complesso nove, perché tre di essi sono stati proposti due volte.

Il test è stato accompagnato da un questionario, composto da due parti principali. La prima conteneva domande legate sia all'indirizzo di studio dei partecipanti sia alle loro precedenti esperienze di apprendimento e acquisizione delle lingue straniere, in particolare dell'italiano. Nella seconda si cercava di sollecitare opinioni personali dei 
soggetti testati riguardo al grado della loro dimestichezza con l'italiano in comparazione con altre lingue straniere, e di indurli a indicare le fonti di acquisizione o apprendimento, evidenziando l'intensità di input linguistici che ne avevano ricavato.

\subsection{Presentazione di alcuni tratti caratteristici dei corsi di laurea frequentati dai par- tecipanti all'esperimento ${ }^{16}$}

La varietà dei corsi nell'ambito della Facoltà di Lettere e Filosofia di Lubiana offre una buona opportunità per la ricerca proposta, visto che l'insegnamento dell'italiano viene impartito sia al Dipartimento di Lingue e letterature romanze - corso di laurea in Lingua e letteratura italiana (Italianistica) ${ }^{17}$ - che nell'ambito del Dipartimento di Traduzione corsi di laurea in Traduzione (Traduzione) e Mediazione interlinguistica (Mediazione) ${ }^{18}$ -, con due approcci in parte diversi, legati alle esigenze dei profili professionali dei futuri laureandi (cioè insegnanti o professionisti nel campo dell'editoria, turismo e simili da una parte e traduttori, interpreti e mediatori interlinguistici dall'altra).

Tutti e tre i corsi hanno in comune l'insegnamento esplicito del funzionamento del sistema verbale italiano a livello morfologico, sintattico e testuale da un lato nonché attività quali ad es. le esercitazioni di traduzione o di composizione di testi dall'altro, nel cui contesto l'attenzione è rivolta principalmente al testo stesso e l'impiego delle forme verbali non costituisce che uno dei tanti aspetti da tenere presenti. Ma da un confronto tra i programmi emerge che gli studenti di Italianistica seguono più lezioni del primo tipo (420) e meno lezioni del secondo (240), mentre per i loro colleghi di Mediazione e di Traduzione la situazione è opposta ( 165 del primo vs 465 del secondo tipo nel caso di Mediazione e 60 del primo vs 600 del secondo tipo nel caso di Traduzione). ${ }^{19}$ Poiché nell'ambito dei corsi di Traduzione e di Mediazione l'italiano è la terza lingua di studio accanto allo sloveno e all'inglese, il numero di lezioni a esso dedicate risulta ridotto (un terzo delle lezioni per il corso di Mediazione, il 25\% per il corso di Traduzione). Il numero di ore obbligatorie dedicate ai vari aspetti dell'italiano (dalla linguistica alla letteratura soprattutto) nell'ambito del corso di Italianistica, invece, raggiunge in complesso 1440 ore nel caso dell'indirizzo pedagogico e 1550 ore nel caso dell'indirizzo non pedagogico.

16 Le informazioni concernenti i corsi di laurea sono state ricavate dai siti internet ufficiali della Facoltà di Lettere e Filosofia dell'Università di Lubiana (http://www.prevajalstvo.net, 7. 4. 2009; http://www.prevajalstvo.net/files/Medjezikovno\%20posredovanje_predstavitveni.pdf, 7. 4. 2009; http://www.ff.uni-lj.si/oddelki/romanistika/IT_star_pravi.htm, 7. 4. 2009).

17 Il corso di laurea in Lingua e letteratura italiana, di durata pari a quattro anni, permette la scelta sia dell'indirizzo pedagogico sia di quello non pedagogico (Miklič/Ožbot 2001: 120).

${ }^{18} \mathrm{Da}$ una decina di anni esiste anche la possibilità di studiare italiano nell'ambito del Dipartimento di Traduzione (Miklič/Ožbot 2001: 120). Sulla scia della riforma dell'istruzione universitaria, il vecchio corso di laurea in Traduzione, di durata pari a 4 anni, è stato da qualche anno sostituito dal Corso di Laurea in Mediazione Interlinguistica (laurea triennale) e da un biennio di specializzazione (Laurea specialistica). I partecipanti all'esperimento del secondo e del terzo anno del Dipartimento di Traduzione erano già iscritti al nuovo indirizzo di studio mentre quelli del quarto seguivano il corso vecchio.

19 I numeri 420, 240, 165, 465, 60, 600 si riferiscono all'insieme delle ore di uno dei due tipi di lezioni offerte agli studenti nell'arco di tutto il corso di laurea. 
Il raffronto dimostra una prevalenza dell'italiano nell'ambito del Dipartimento di lingue e letterature romanze. Tuttavia, il corso di laurea in Italianistica si accompagna obbligatoriamente a un altro corso di laurea di pari livello, che non necessariamente si colloca nell'ambito dello studio delle lingue; il fatto che, invece, gli studenti del Dipartimento di Traduzione studino contestualmente l'inglese e lo sloveno potrebbe contribuire a determinare in loro una maggiore sensibilità per il fatto linguistico.

La domanda che mi sono posta come guida della mia indagine è se sia possibile raggiungere lo stesso grado di competenza nell'uso delle forme del sistema verbale italiano anche con un numero di lezioni dedicate all'apprendimento sistematico ed esplicito delle strutture della lingua italiana decisamente inferiore e con più diffuse attività che concernono la produzione di testi, da cui gli studenti potrebbero acquisire competenze per via induttiva. Va, però, aggiunto che con il presente contributo non si intende privilegiare uno dei due approcci a scapito dell'altro visto che le differenze sono in gran parte giustificate dagli scopi educativi.

\subsection{Caratteristiche del campione}

Ho deciso di sottoporre al test gli studenti del $2^{\circ}, 3^{\circ}$ e del $4^{\circ}$ anno del corso di Italianistica, gli studenti del $2^{\circ}$ e del $3^{\circ}$ anno di Mediazione e quelli del $4^{\circ}$ anno di Traduzione..$^{20}$

\begin{tabular}{|c|c|c|c|c|c|c|c|}
\hline \multicolumn{4}{|c|}{ Corso di lingua e letteratura italiana } & \multicolumn{2}{|c|}{ Corsi di Traduzione e di Mediazione interlinguistica } \\
\hline $\begin{array}{c}\text { Partecipanti } \\
\text { del } \\
2^{\circ} \text { anno del } \\
\text { corso di } \\
\begin{array}{c}\text { Italianistica } \\
\text { (IT2) }\end{array}\end{array}$ & $\begin{array}{c}\text { Partecipanti } \\
\text { del } \\
3^{\circ} \text { anno del } \\
\text { corso di } \\
\text { Italianistica } \\
\text { (IT3) }\end{array}$ & $\begin{array}{c}\text { Partecipanti } \\
\text { del } \\
4^{\circ} \text { anno del } \\
\text { corso di } \\
\text { Italianistica } \\
\text { (IT4) }\end{array}$ & $\begin{array}{c}\text { Numero com- } \\
\text { plessivo } \\
\text { di tutti i } \\
\text { partecipanti } \\
\text { del corso di } \\
\text { Italianistica } \\
\text { (IT) }\end{array}$ & $\begin{array}{c}\text { Partecipanti } \\
\text { del } \\
2^{\circ} \text { anno } \\
\text { del corso di } \\
\text { Mediazione } \\
\text { (TRA2) }\end{array}$ & $\begin{array}{c}\text { Partecipanti } \\
\text { del } \\
3^{\circ} \text { anno } \\
\text { del corso di } \\
\text { Mediazione } \\
\text { (TRA3) }\end{array}$ & $\begin{array}{c}\text { Partecipanti } \\
\text { del } \\
4^{\circ} \text { anno del } \\
\text { corso di } \\
\text { Traduzione } \\
\text { (TRA4) }\end{array}$ & $\begin{array}{c}\text { Numero com- } \\
\text { plessivo } \\
\text { di tutti i } \\
\text { partecipanti } \\
\text { dei corsi di } \\
\text { Traduzione e } \\
\text { Mediazione } \\
\text { (TRA) }\end{array}$ \\
\hline 12 & $12 / 11^{21}$ & 12 & $36 / 35$ & 14 & 12 & 9 & 35 \\
\hline
\end{tabular}

Tabella 1: Numero dei partecipanti22

Gli studenti del $2^{\circ}, 3^{\circ}$ e del $4^{\circ}$ anno di IT nonché gli studenti del $4^{\circ}$ anno di TRA erano al corrente delle tematiche su cui si incentrava la ricerca. Il $4^{\circ}$ anno IT si distingueva per aver ricevuto la quantità maggiore di insegnamento esplicito del funzionamento del sistema verbale italiano a livello morfologico, sintattico e testuale. Gli studenti del $2^{\circ}$ anno IT, invece, seppur a conoscenza di tutti i fenomeni dal punto di vista teorico, possedevano una scarsa esperienza pratica. Gli studenti del $2^{\circ}$ e del $3^{\circ}$ anno TRA, infine,

${ }^{20}$ Siccome mi ero proposta di riflettere sull'insegnamento esplicito degli usi delle forme verbali italiane a livello universitario la partecipazione del $1^{\circ}$ anno, dopo pochi mesi di esposizione a lezioni di questo tipo, non avrebbe contribuito a una maggiore oggettività dei risultati.

21 Alla prima parte del test hanno partecipato 12 studenti, alla seconda, invece, 11 soltanto.

22 Il numero dei partecipanti di ogni singolo gruppo non coincide con il numero complessivo degli studenti della stessa classe, visto che il test non era stato annunciato in anticipo. Sono stati coinvolti, quindi, gli studenti venuti con l'intenzione di partecipare alla lezione regolare di quel giorno. 
avevano appreso soltanto una parte delle tematiche. In comparazione con gli altri gruppi, il $2^{\circ}$ anno TRA aveva subito la quantità minore di insegnamento esplicito.

A questo punto bisogna completare il quadro relativo al campione anche con alcuni dati ricavati dal questionario. Per quel che riguarda il punteggio ottenuto all'esame di maturità, che permetterebbe un paragone a livello nazionale, e i voti universitari dei partecipanti, entrambi indicatori sia delle capacità intellettuali che dell'atteggiamento verso lo studio, i gruppi presentano caratteristiche affini. Mentre la media dei punti all'esame di maturità mostra un lieve vantaggio degli studenti di Mediazione e di Traduzione (21,53 vs 20,4) in rapporto all'altro gruppo, la media dei voti universitari si rivela a favore degli studenti di Italianistica (7,87 vs 7,85). Anche se la maggioranza riteneva che al momento dell'iscrizione all'università padroneggiasse bene l'italiano e pochi soltanto si dichiaravano principianti, la valutazione di alcuni altri parametri ${ }^{23}$ ha rivelato un grado di preconoscenze superiore negli studenti di Traduzione e di Mediazione. ${ }^{24}$

\subsection{Classificazione delle soluzioni}

Nella fase iniziale dell'analisi ho suddiviso le soluzioni degli studenti in tre sottogruppi: soluzioni corrette (corr.), soluzioni parzialmente corrette (pcorr.), soluzioni inappropriate (err.). Si sono classificati tra le soluzioni parzialmente corrette gli errori di tipo ortografico (incomincio invece di incominciò), morfologico (eravano invece di erano), morfosintattico (avevano partito invece di erano partiti), lessicale (tirata invece di trainata) e le omissioni di elementi senza ripercussioni sull'uso appropriato delle forme verbali (aveva fatto invece di aveva più fatto), mentre hanno trovato posto nel secondo sottogruppo gli errori di tipo sintattico (la segnalazione non-appropriata dei rapporti temporali: giurò invece di aveva giurato per l'anteriorità nel passato ecc.), semantico (mancata comprensione del contenuto: significherebbe invece di significa ecc.), sintattico-pragmatico (difficoltà di scelta tra il perfetto e l'imperfetto: fu determinato invece di era determinato; scelta del passato prossimo (ha cominciato) al posto del passato remoto (incominciò) per presentare azioni centrali in un testo dal contenuto storico ecc.), nonché gli spazi da colmare lasciati vuoti, presumibilmente a causa di difficoltà semantiche o sintattiche. Ho deciso di elaborare una tale suddivisione perché soltanto gli errori del secondo tipo riflettono difficoltà di comprensione e d'uso. ${ }^{25}$

${ }^{23}$ Sono stati considerati gli anni dell'apprendimento scolastico, la scelta dell'italiano come materia da studiare per l'esame di maturità, le fonti più importanti dell'acquisizione e dell'apprendimento, i contatti con la lingua, il paragone delle competenze in italiano rispetto alle competenze in inglese $o$ in un'altra lingua straniera.

${ }^{24} \mathrm{Si}$ alternano ai primi tre posti i gruppi TRA2, TRA3 e IT4, con prevalenza dei primi due, al quarto posto si collocano soprattutto studenti del gruppo IT3, mentre agli ultimi due posti troviamo i gruppi TRA4 e IT2.

25 Spesso un'unica soluzione conteneva non una sola, bensì più inesattezze. Se presentava una combinazione di imprecisioni del primo gruppo, è stata unita alle soluzioni parzialmente corrette. Se, invece, presentava, accanto a uno o più errori del primo gruppo, un aspetto qualsiasi del secondo, è stata classificata tra le soluzioni errate. Similmente sono state considerate errate anche le soluzioni caratterizzate da una combinazione di errori del secondo tipo. 


\subsection{Classificazione dei fenomeni osservati in base alla quantità di risposte inappro- priate}

La Tabella 5 (per la sua lunghezza è stata inserita nell'Appendice) riassume i dati principali delle analisi. È stata elaborata in modo tale da disporre le soluzioni degli studenti in un ordine che tiene conto del grado di criticità di ciascun fenomeno.

La Tabella 5 mette in evidenza un fatto molto curioso: pare che lo stesso fenomeno possa presentare livelli diversi di criticità (si osservino gli esempi di flash-back e di azioni extratemporali). Si potrebbe, dunque, sostenere che la valutazione del mondo testuale sottostante e degli elementi di cotesto in cui il fenomeno è inserito sia decisiva nella scelta appropriata dei mezzi linguistici.

\subsection{Mondo testuale e cotesto}

L'analisi dei risultati dimostra che gli studenti hanno capito relativamente bene il mondo testuale, ossia i contenuti di ogni singolo testo. Infatti, hanno ritenuto sconosciuto soltanto $1{ }^{\prime} 1,04 \%$ di tutte le espressioni usate nei testi. ${ }^{26}$ La quantità di errori di tipo semantico, inoltre, si è rivelata molto inferiore rispetto agli errori di tipo sintattico o sintatticopragmatico. Alcuni altri dati svelano qualche difficoltà in più: dovendo tradurre il testo D in sloveno gli studenti si sono resi conto delle difficoltà semantiche e lessicali in misura maggiore di quanto non fosse accaduto nella fase che prevedeva la sola identificazione di espressioni sconosciute nel cloze B. Una percentuale superiore al 60\% - il 61,11\% degli studenti di Italianistica e il $62,86 \%$ di quelli di Traduzione - ha dichiarato di non aver avuto difficoltà a capire il contenuto del cloze GFC, il più complicato di tutti. Difficoltà in tal senso sono state evidenziate dal 33,33\% degli studenti di Italianistica e dal 37,14\% di quelli di Traduzione. Va notato il fatto che il gruppo IT2 ha risolto con un maggior numero di forme appropriate i cloze preceduti dalla versione slovena (2E, 2GFC, C, 2D) del testo; ciò si è verificato in misura minore anche per i gruppi TRA4 e TRA3. È, quindi, possibile che il motivo per cui il gruppo IT2 si è classificato all'ultimo posto della graduatoria generale (si veda 2.9) sia in parte imputabile alle difficoltà di comprensione. $\mathrm{Nel}$ complesso i cloze preceduti dalla versione slovena non sono caratterizzati sistematicamente da una quantità superiore di soluzioni appropriate rispetto agli altri cloze, il che potrebbe essere interpretato come segno di assenza di difficoltà significative degli studenti nel comprendere il mondo testuale. Il paragone tra i risultati dei cloze $\mathrm{D}, \mathrm{E}, \mathrm{GFC}$ e i rispettivi cloze 2D, 2E, 2GFC - che riflettono la prestazione degli studenti prima e dopo il confronto con la versione slovena - ha, tuttavia, rivelato quasi sempre un miglioramento della prestazione successiva alla lettura o all'elaborazione della versione slovena.

Una delle strategie basilari che aiutano a riempire il cloze con le forme appropriate, considerando il contenuto del testo come un'unità e non solo sulla base degli elementi di cotesto vicino, consiste nella prelettura. Nel caso dei testi brevi della prima parte del test il 68,57\% degli studenti ha asserito di aver letto gli interi cloze prima di

\footnotetext{
${ }^{26}$ In condizioni ottimali il lettore ha bisogno, all'interno di un testo, di conoscere il $95 \%$ del lessico per poter ricreare con successo il mondo testuale. Il rimanente $5 \%$ può essere desunto dal contesto (Šifrar Kalan 2008: 53).
} 
cominciare a inserire ${ }^{27}$ le forme, il $22,86 \%$, invece, ha iniziato a risolvere l'esercizio senza una prelettura. Nel caso del testo GFC - più lungo e complesso e meno completo - solo il 58,57\% degli studenti ha letto interamente il cloze prima di inserire le soluzioni, ${ }^{28}$ il $30 \%$, invece, non lo ha fatto. La sola prelettura, tuttavia, non ha comportato necessariamente un risultato migliore. ${ }^{29} \mathrm{E}$ importante, quindi, che gli studenti imparino a osservare appropriatamente gli elementi di cotesto e ad accedere per mezzo di questi elementi ai contenuti.

Nell'argomentare le proprie scelte circa il 20\% degli studenti chiama in causa gli elementi di cotesto (una percentuale maggiore, invece, basa la propria scelta sull'individuazione dei rapporti temporali tra le azioni). Questa tendenza è un po' più forte tra gli studenti di Traduzione. Tali argomentazioni mostrano spesso un ragionamento inappropriato o superficiale da parte degli studenti, ma in altri casi ci fanno notare che essi si sono serviti con successo del cotesto per identificare il paradigma con cui denotare azioni centrali. Questa tattica, tuttavia, non è stata applicata sempre e da tutti; soprattutto gli studenti di Italianistica avrebbero potuto fare meno errori con un'osservazione più attenta del cotesto. ${ }^{30}$ Un'osservazione più attenta avrebbe potuto eliminare anche alcuni errori di tipo ortografico e morfologico.

\subsection{Interferenza e coincidenza}

Le conseguenze di un'insufficiente osservazione degli elementi sopra menzionati e un sapere non abbastanza consolidato circa gli usi delle forme verbali italiane si manifestano nelle scelte influenzate dall'interferenza, soprattutto della prima lingua, cioè dello sloveno. L'esito delle analisi sembra suggerire che l'interferenza si manifesti a due livelli principali: al livello delle forme verbali ${ }^{31}$ e al livello delle strutture di superficie. I casi di interferenza sono stati classificati in cinque gruppi: i primi quattro riguardano il primo livello, mentre l'ultimo si riferisce al secondo.

27 75\% (IT2), 75\% (IT3), 91,67\% (IT4), 57,14\% (TRA2), 50\% (TRA3), 55,56\% (TRA4).

$2850 \%$ (IT2), 54,55\% (IT3), 83,33\% (IT4), 50\% (TRA2), 50\% (TRA3), 66,67\% (TRA4).

${ }^{29}$ Mentre nel caso del gruppo IT4 effettivamente esiste una correlazione positiva tra i due fenomeni, il confronto dei risultati dei gruppi TRA3 e IT2 mette in luce una situazione opposta: anche se un numero maggiore di studenti aveva letto i cloze in precedenza, il gruppo IT2 non ha ottenuto buoni risultati; d'altro lato il gruppo TRA3, che in misura minore si era servito della preletura, ha ottenuto risultati assai migliori.

${ }^{30}$ Nell'insegnamento la scelta del paradigma centrale da inserire nel cloze non deve essere limitata soltanto all'osservazione del cotesto, ma devono essere spiegate agli studenti le ragioni più profonde per cui una tale scelta risulta appropriata. Uno degli elementi che può incidere sulla scelta consiste, ad es., nel genere testuale.

31 In questo senso il sedanjik («presente sloveno») trova la forma «corrispondente» nel presente dell'indicativo, il pogojnik («condizionale sloveno») nel condizionale semplice, il dovršnik preteklika («preterito del verbo perfettivo sloveno») nel passato prossimo o nel passato remoto, il nedovršnik preteklika («preterito del verbo imperfettivo sloveno») nell'imperfetto dell'indicativo e il prihodnjik («futuro sloveno») nel futuro semplice. 
a) Interferenza totale dello sloveno $\left(\mathrm{s}^{+}\right)$

(1) Al giudice che gli chiese come mai AVESSE ASPETTATO/AVEVA ASPETTATO tanto a denunciare la scomparsa, il marito abbandonato rispose.

(2) Al giudice che gli chiese come mai *ASPETTAVA tanto a denunciare la scomparsa, il marito abbandonato rispose.

L'interferenza, riflessa nell'uso dell'IM aspettava al posto del TP'/TP avesse aspettato/aveva aspettato nei test di alcuni studenti, si manifesta in due punti: influenzati dallo sloveno, che nelle completive segnala l'anteriorità nel passato con il preteklik e non con il predpreteklik (il trapassato sloveno), alcuni studenti non hanno scelto il TP neppure nel testo italiano. La scelta dell'IM (e non del PR) è inoltre dovuta all'influsso della forma imperfettiva del verbo sloveno (je čakal, PRET-ND).

b) Interferenza parziale dello sloveno (s/)

(3) Al giudice che gli chiese come mai *ASPETTÒ tanto a denunciare la scomparsa, il marito abbandonato rispose...

Nel caso dell'impiego del PR aspettò non è stata segnalata l'anteriorità, ma a differenza del caso precedente, il ND, con il suo carattere imperfettivo, non ha esercitato alcuna influenza sulla scelta del paradigma italiano.

c) Interferenza totale dello sloveno e dell'italiano del Nord d'Italia (sn+)

(4) Tempo fa, il signor Norman Long di Saint Petersburg, una città della Florida, intentò una causa di divorzio contro la moglie Lizzie, accusandola di abbandono del tetto coniugale [...] Al giudice che gli *HA CHIESTO come mai avesse aspettato tanto a denunciare la scomparsa, il marito abbandonato rispose... (sn+)

Con l'impiego del PP gli studenti non hanno rispettato la scelta dell'autore del testo di presentare le azioni centrali (tra cui anche chiedere) per mezzo del PR. A causa della forma perfettiva del verbo je vprašal (PRET-D) gli studenti non hanno esitato ad usare il PP (paradigma perfettivo): l'esempio si è classificato, quindi, tra i casi di interferenza totale.

Negli studenti si è potuta spesso notare la tendenza ad usare il $\mathrm{PP}^{32}$ nelle situazioni che in sloveno richiederebbero il preteklik. Il fenomeno è probabilmente dovuto al fatto che nell'apprendimento scolastico il PP viene insegnato relativamente presto rispetto al PR, all'IM e al TP. Gli studenti tendono ad usare il PP più del necessario anche per la sua forte presenza - rispetto al PR - nell'italiano del Nord d'Italia, zona confinante con la Slovenia.

d) Interferenza parziale dello sloveno e dell'italiano del Nord d'Italia (sn/)

(5) Mi bastò entrare nella sua stanza, invece, (dopo avermi introdotto, Perotti aveva richiuso discretamente la porta dietro le mie spalle, per vedere che mi sorrideva benigna, gentile, amica. Ancor più dell'esplicito invito a venire avanti, * $\underline{\text { È STATO }}$ quel suo sorriso luminoso, pieno di tenerezza e di perdono, che mi persuase a staccarmi dal fondo buio della stanza e ad avvicinarmi.

32 Questa tendenza è più forte nel gruppo IT2 (73 casi), che è seguito dai gruppi TRA2 (49), IT3 (38), TRA3 (30), TRA4 (20) e IT4 (17). 
Con l'uso del PP gli studenti hanno rispettato la necessità di presentare l'azione globalmente e non si sono lasciati influenzare dalla forma imperfettiva (ND) del verbo essere sloveno, ma, per ragioni simili a quelle del caso precedente, non hanno usato il PR.

e) Interferenza causata dalle strutture di superficie del testo sloveno $\left(\mathrm{st}^{+}\right)^{33}$

(6) Proti koncu leta 1510 je papež Julij II začel razkazovati svojo gosto brado, nad katero SO SE ZGRAŽALI kardinali in poslaniki.

(7) Verso la fine del 1510, papa Giulio II incominciò a sfoggiare una folta barba, che SCANDALIZZAVA cardinali e ambasciatori.

(8) Verso la fine del 1510, papa Giulio II incominciò a sfoggiare una folta barba, che *SCANDALIZZARONO/*SCANDALIZZAVANO/*E STATA SCANDALIZZATA cardinali e ambasciatori.

Influenzati dal sintagma verbale so se zgražali nad nella traduzione slovena del testo C (da cui quest'ultimo era preceduto) che richiede il sostantivo barba in funzione di oggetto preposizionale, e il sintagma nominale cardinali e ambasciatori in funzione di soggetto, alcuni studenti hanno proposto come soluzione paradigmi quali scandalizzarono, scandalizzavano, è stata scandalizzata, evidentemente pensando che anche con il verbo scandalizzare le entità barba e cardinali e ambasciatori dovessero svolgere la funzione rispettivamente di oggetto e soggetto, o almeno ricoprire gli stessi ruoli semantici, cioè quello di tema nel primo, e quello di agente nel secondo caso. ${ }^{34}$

In alcuni casi la soluzione proposta dagli studenti sembra suggerire una loro interpretazione diversa del mondo testuale, che in sloveno sarebbe verbalizzata per mezzo di un paradigma considerato analogo alla variante scelta. Ma dato che non avevo una conferma più solida, ho potuto soltanto ipotizzare l'interferenza. Ho denominato questo tipo di interferenza interferenza probabile (+?, /?). Si osservi il caso dell'azione in D.3:

(9) Il biblico patriarca Giacobbe decise che al suo ultimo figlio avrebbe dato il nome augurale di Ben Yamin (Beniamino), che in ebraico SIGNIFICA «figlio della destra», e dunque "fortunato», poiché la sinistra_VENIVA considerata infausta.

(10) Svetopisemski očak Jakob se je odločil, da bo svojemu zadnjemu sinu za dobrodošlico dal ime Ben Yamin (Benjamin), kar v hebrejščini POMENI »sin desnice« in zatorej »srečen«, saj JE levica VELJALA za nesrečno.

A differenza delle azioni decise (PR) e avrebbe dato (CC), l'azione significa (Pr) non si trova nella sfera del passato bensì nell'extratemporalità. Nel testo sloveno sarebbe perciò verbalizzata per mezzo del SED. D'altro canto l'azione veniva (IM), simultanea all'azione decise,

$33 \mathrm{Ci}$ sono anche dei casi caratterizzati da una combinazione ulteriore dei quattro principali tipi di interferenza, ad. es. interferenza combinata dello sloveno e dell'italiano del Nord d'Italia e delle strutture di superficie del testo sloveno $\left(\mathrm{snt}^{+}\right)$.

34 Un esempio simile è rappresentato dalle soluzioni influenzate dalla diatesi attiva in sloveno, quali chiamino, chiamarono, chiamassero, chiamerebbe, chiamasse in punto D.6, che in italiano invece richiede l'impiego della diatesi passiva. 
appartiene al passato. Nel testo sloveno sarebbe verbalizzata per mezzo del PRET-ND. Si tratta, dunque, di una situazione di coincidenza che già di per sé avrebbe potuto comportare l'uso dell'IM. Ma invece dell'IM alcuni hanno proposto il Pr (viene):

(11) Il biblico patriarca Giacobbe decise che al suo ultimo figlio avrebbe dato il nome augurale di Ben Yamin (Beniamino), che in ebraico SIGNIFICA «figlio della destra», e dunque «fortunato», poiché la sinistra *VIENE considerata infausta.

Con molta probabilità la loro scelta è dovuta a una diversa interpretazione della posizione temporale dell'azione veniva: anche quest'ultima è stata interpretata come extratemporale per l'attrazione esercitata dall'azione extratemporale significa e a causa della presenza del binomio destra - sinistra. Ciò ha comportato l'uso del Pr (viene). Visto che in una situazione del genere anche in sloveno sarebbe stato usato il SED (saj levica ${ }^{*}$ velja za nesrečno) si ha di nuovo un caso di coincidenza. Ma siccome questa interpretazione risulta inadeguata a rendere il significato completo della frase (il fatto che la sinistra veniva considerata infausta giustifica la scelta del nome), ho classificato il caso tra i casi di interferenza probabile e non tra i casi di coincidenza.

Servendomi di questa suddivisione ho poi elaborato una nuova classificazione dei casi di interferenza, distinguendo tra casi di interferenza totale ${ }^{35}(+)$, casi di interferenza parziale $(/),{ }^{36}$ e casi di interferenza probabile $(+?, /$ /?). L'interferenza totale determina 32 su 65 casi di soluzioni inappropriate con la maggiore frequenza di occorrenze (si vedano i dati presentati nella Tabella 5). Se si considerano anche le soluzioni più frequenti con interferenza parziale, ci si arriva a 37 casi. Se a questi si aggiungono anche i casi di interferenza probabile, i casi sono $41 \mathrm{su} 65$. I fenomeni di interferenza compaiono con una percentuale lievemente superiore tra gli studenti di Traduzione.

\begin{tabular}{|l|c|c|c|c|c|c|c|c|}
\hline Tipo di interferenza & IT2 & IT3 & IT4 & IT & TRA2 & TRA3 & TRA4 & TRA \\
\hline$(+)$ & $20,17 \%$ & $14,53 \%$ & $10,87 \%$ & $15,19 \%$ & $18,7 \%$ & $17,16 \%$ & $15,25 \%$ & $17,04 \%$ \\
\hline$(+),()$ & $23,39 \%$ & $18,71 \%$ & $13,67 \%$ & $18,59 \%$ & $23,44 \%$ & $21,71 \%$ & $20,23 \%$ & $21,79 \%$ \\
\hline$(+),(),(?)$ & $26,82 \%$ & $20,85 \%$ & $16,1 \%$ & $21,26 \%$ & $25,8 \%$ & $24,78 \%$ & $21,8 \%$ & $24,13 \%$ \\
\hline
\end{tabular}

Tabella 2: Percentuale media di soluzioni determinate dall'interferenza

Gli studenti del $4^{\circ}$ anno di Italianistica risultano decisamente i meno soggetti al fenomeno dell'interferenza; li seguono, nell'ordine, gli studenti del $3^{\circ}$ anno di Italianistica e del $4^{\circ}$ anno di Traduzione, quelli del $3^{\circ}$ anno di Mediazione, quelli del $2^{\circ}$ anno di Mediazione e del $2^{\circ}$ anno di Italianistica. In linea di massima l'interferenza diminuisce con il progredire degli studi, con un'intensità maggiore nel gruppo di Italianistica (si noti il dislivello notevole tra l'IT4 e l'IT2) e minore nel gruppo di Traduzione e di

35 Sono stati presi in considerazione i casi di interferenza, caratterizzati dai simboli $\mathrm{s}^{+}, \mathrm{sn}^{+}, \mathrm{st}^{+}, \mathrm{snt}^{+}$. ${ }^{36} \mathrm{Si}$ tratta dei casi di interferenza, caratterizzati dai simboli sn/ e s/. 
Mediazione che si rivela più omogeneo, ma anche un po' meno segnato da progressi in itinere. È da notare la posizione del $3^{\circ}$ anno di Mediazione che si classifica piuttosto male rispetto al secondo posto nella classifica generale (si veda 2.9). Il risultato migliore del gruppo IT (si tratta, tuttavia, di differenze minime) potrebbe essere collegato all'insegnamento esplicito degli aspetti morfosintattici e degli usi dei paradigmi verbali in chiave contrastiva e testuale. D'altro lato le scelte compiute dagli studenti nei punti di coincidenza ${ }^{37}$ dimostrano che gli studenti - in occasioni in cui avrebbero potuto farlo - non sempre hanno proposto forme verbali ritenute analoghe. ${ }^{38}$

(12) Secondo quanto afferma l'ingegner Felice Vinci nel libro Omero nel Baltico, le gesta dei Danai, narrate nei poemi omerici, non si sarebbero svolte nel Mediterraneo, ma nei mari del Nordeuropa. Partendo da uno scritto di Plutarco, nel quale lo storico greco sostiene che l'isola di Calipso, Ogigia, è situata a cinque giorni di navigazione dalla Britannia, Vinci ha analizzato numerosi toponimi dell'Europa settentrionale, sorprendentemente simili a quelli omerici, nonché svariati fenomeni naturali, come gli enormi gorghi d'acqua detti maelstrom, che RICORDANO (Pr) Scilla e Cariddi. In sloveno: $k i$ SPOMINJAJO (SED-ND) na Scilo in Karibdo.

Soluzioni inappropriate proposte dagli studenti: *ricordavano, *ricordava, *ricordassero, *hanno ricordato, * ricordarono.

Il confronto tra le percentuali di soluzioni appropriate nei casi di coincidenza e la percentuale media di tutte le soluzioni appropriate del rispettivo esercizio evidenzia una prestazione migliore nei casi di coincidenza da parte del del gruppo TRA, mentre il gruppo IT è meno incline a scegliere i paradigmi secondo le analogie.

\subsection{Commento dei punti critici}

\subsubsection{Azioni anteriori verbalizzate nell'ambito delle frasi indipendenti: flash-back}

Mentre nei testi sloveni, nella narrazione dei fatti passati, le azioni omesse in un primo tempo vengono recuperate tramite l'uso dello stesso paradigma con cui sono denotate le azioni nel fuoco narrativo, per cui il rapporto di anteriorità deve essere dedotto soltanto dagli elementi di co- e contesto, in quelli italiani tali azioni sono esplicitate anche per mezzo di un paradigma in grado di segnalare l'anteriorità.

I cloze proposti presentavano 7 casi di flash-back: in 5 di questi si è raggiunta una percentuale molto elevata (oltre 1'80\%) di soluzioni inappropriate. Gli studenti di

${ }^{37}$ Con il termine coincidenza sono indicati i casi in cui il testo italiano e la corrispettiva traduzione in sloveno richiedono l'uso dei paradigmi ritenuti analoghi nell'immaginario laico di un parlante sloveno. A differenza di quanto accade nei casi di interferenza, nei casi di coincidenza la scelta della forma ritenuta analoga è quella appropriata.

${ }^{38} \mathrm{Si}$ osservi la posizione nella classifica dei casi (Tabella 5): A.3, B.3, C.1, C.2, C.6, C.10, D.2, 2D.2, D.3, 2D.3, D.4, 2D.4, E.2, 2E.2, E.4, 2E.4, E.5, 2E.5, E.7, 2E.7, GFC.4, 2GFC.4, GFC.6, 2GFC.6, GFC.7, 2GFC.7. 
Italianistica in 5 casi hanno ottenuto risultati migliori rispetto ai colleghi di Traduzione. ${ }^{39}$ Tra le soluzioni inappropriate spiccano quelle evidentemente influenzate dall'interferenza con lo sloveno: mentre il PRET-ND ha suggerito la scelta dell'IM, il PRET-D ha provocato l'impiego del PR o del PP nel testo italiano. Si osservi l'uso dei paradigmi verbali in GFC.5 e GFC.8:

(13) Mi bastò entrare (PR) nella sua stanza [...] per vedere che mi sorrideva benigna, gentile, amica [...] Mi accostai (PR) dunque al letto, dalla parte dei piedi [...] Benché infilata sotto le coperte, Micòl ne rimaneva fuori con tutto il busto [...] Quando ${ }^{*}$ ENTRAI (PR)/*SONO ENTRATO (PP) (GFC.5) stava leggendo (IM): un romanzo francese, come notai, riconoscendo di lontano il tipo della copertina bianca e rossa; ed *ERA (IM) (GFC.8) la lettura, probabilmente, piú che il raffreddore, a metterle (INF) sotto gli occhi un segno di stanchezza.

In sloveno: sem vstopil (PRET-D) in GFC.5; je bila (PRET-ND) in GFC.8.

Soluzioni appropriate: ero entrato (TP) in GFC.5; era stata (TP) in GFC.8.

Dal confronto dei 7 brani in cui appaiono i casi di FB emerge il ruolo decisivo, nel processo di individuazione del fenomeno, della complessità sintattica, lessicale, stilistica, contenutistica nonché del fattore della lunghezza del testo. Sono questi i motivi per cui i FB del testo GFC sono stati così difficili da decifrare. I casi B.1 e B.2, legati per mezzo della coordinazione copulativa, mettono in evidenza l'influsso della distanza dalla precedente azione nel fuoco narrativo sull'individuazione del fenomeno: mentre il FB del B.1 (si era allontanata) è stato riconosciuto dal 52,11\% degli studenti, il FB del B.2 (aveva più fatto ritorno) è stato segnalato soltanto nel 18,31\% dei casi:

(14) Tempo fa, il signor Norman Long di Saint Petersburg, una città della Florida, intentò (PR) una causa di divorzio contro la moglie Lizzie, accusandola di abbandono del tetto coniugale. La donna SI ERA ALLONTANATA (TP)/*SI ALLONTANÒ (PR) (B.1) da casa poco dopo le nozze, celebrate più di quarant'anni prima, e NON vi AVEVA più FATTO (TP)/*FECE (PR)/*HA FATTO (PP) (B.2) ritorno. Al giudice che gli chiese (PR) come mai ...

L'esempio A.4 rappresenta, per la chiarezza dei contenuti e delle strutture sintattiche del primo testo, il caso con il maggior numero di soluzioni esatte (71,82\%):

(15) I soccorritori li hanno raggiunti (PP) solo dopo un certo tempo, ma i tre erano (IM) già fuori pericolo: il cane li AVEVA ESTRATTI (TP)/*HA ESTRATTO/*HA ESTRATTI/*È STATO ESTRATTO (PP) dalla neve.

${ }^{39}$ Nell'ambito di Italianistica il gruppo IT4 è stato il migliore nell'identificazione dei FB (33,33\%), seguito dai gruppi IT3 $(26,51 \%)$ e IT2 $(17,86 \%)$, mentre fra gli studenti del gruppo TRA il miglior risultato è stato ottenuto dal gruppo TRA2 (21,43\%), seguito dai gruppi TRA3 $(20,24 \%)$ e TRA4 $(19,05 \%)$. 


\subsubsection{Azioni anteriori nel passato verbalizzate nell'ambito della dipendente relativa}

L'espressione dell'anteriorità nell'ambito delle dipendenti relative, sia in sloveno che in italiano, è analoga all'espressione del FB, per cui presenta anche lo stesso tipo di difficoltà:

(16) Dejansko pa je na ta način papež želel (PRET-ND) posnemati Julija Cezarja, ki JE leta 54 pr. Kr. PRISEGEL (PRET-D), da se ne bo obril, dokler ne bo maščeval smrti svojih mož, ki so jih pomorili Galci.

(17) In realtà, con tale gesto il Papa intendeva (IM) emulare Giulio Cesare, il quale nel 54 a.C. AVEVA GIURATO (TP)/* GIURÒ (PR)/*HA GIURATO (PP) $/{ }^{*}$ GIURAVA (IM)/*EBBE GIURATO (TR) di non radersi finché non avesse vendicato la morte dei propri uomini massacrati dai Galli.

Tutti e tre i casi caratterizzati da questo fenomeno (C.7, D.5, 2D.5) presentano oltre il $50 \%$ di soluzioni inappropriate. Il risultato migliore (le soluzioni con il TP) è stato di nuovo ottenuto dal gruppo IT (IT: 50,93\%; TRA: 33,43\%). Il confronto tra gli studenti all'interno di ciascuno dei due gruppi IT e TRA mette in luce un fatto curioso: i due sottogruppi migliori della classifica generale, l'IT4 e il TRA3 (si veda 2.9), hanno ottenuto il risultato meno buono. ${ }^{40} \mathrm{Tra}$ le soluzioni inappropriate prevalgono quelle dettate dall'influsso dello sloveno, con un'intensità maggiore negli studenti di traduzione (IT: $41,67 \%$; TRA: $51,43 \%{ }^{41}$ ).

\subsubsection{Espressione della temporalità relativa nel passato nell'ambito delle completive}

L'impiego delle forme verbali nelle completive ha presentato gradi diversi di criticità. ${ }^{42}$ La più critica si è rivelata - a causa della complessità del costrutto sintattico, caratterizzato da una temporale di secondo grado nell'ambito della completiva implicita - l'espressione dell'anteriorità nella posteriorità nel passato:43

(18) Po besedah kronista iz Bologne je bil Julij II odločen (PRET-ND), da si pusti (SEDD) rasti brado, dokler NE BO PREMAGAL IN PREGNAL (PRIH-D) iz Italije francoskega kralja Ludvika XII.

(19) Secondo quanto riportato da un cronista bolognese, Giulio II era determinato (IM) a farsi crescere (INF) la barba fino a quando NON AVESSE SCONFITTO E CACCIATO (TP') dall'Italia il re francese Luigi XII.

La percentuale media di soluzioni inappropriate in C.11 e C.8 è del 64,79\%; esse risultano prevalenti nel gruppo TRA (IT: 59,72\%; TRA: 70\%). ${ }^{44}$ A prima vista

40 Soluzioni con il TP: 55,55\% (IT2, IT3), 41,67\% (IT4), 35,71\% (TRA2), 25\% (TRA3) 33,33\% (TRA4).

41 Sono stati presi in considerazione sia i casi di interferenza totale che quelli di interferenza parziale. Tale tipo di errori costituisce il 57,14\% (TRA2), il 55,56\% (IT3 e TRA4), il 41,67\% (TRA3), il $36,11 \%$ (IT4) e il 33,33\% (IT2) di tutte le soluzioni.

$42 \mathrm{Si}$ osservi nella Tabella 5 la posizione dei casi B.4, B.5, C.7, D.1, 2D.1, D.6, 2D.6, GFC.2, 2GFC.2.

43 Per una discussione più approfondita si veda Miklič (1991: 100).

44 Usi appropriati (TP'/IM'): 45,83\% (IT2), 54,17\% (IT3), 20,83\% (IT4), 28,57\% (TRA2), 25\% (TRA3), $38,89 \%$ (TRA4). 
potrebbe sorprendere il fatto che la quantità più elevata di soluzioni inadeguate sia stata prodotta dai due gruppi migliori (IT4 e TRA3), ma dall'analisi delle motivazioni della scelta addotte da coloro che hanno inserito una delle due soluzioni appropriate, il TP' (avesse vendicato, non avesse sconfitto e cacciato) o l'IM' (vendicasse, non sconfiggesse e cacciasse), si può evincere che tale scelta è frutto non di un esame consapevole dei rapporti temporali tra le azioni - supportato da competenze attive nell'uso dei paradigmi verbali - ma di automatismo o casualità. Nell'argomentare la scelta soprattutto del congiuntivo, infatti, molti hanno chiamato in causa la congiunzione finché, che di per sé non determina il suo uso. Si sono avvicinati di più all'interpretazione corretta dei rapporti temporali tra le azioni gli studenti che hanno proposto come soluzione il $\mathrm{CC}^{45}$ pensando, forse anche per non aver notato la completiva implicita, che si trattasse di una semplice posteriorità nel passato. Gli usi del F, Pr, FF e C sono dovuti all'interferenza dello sloveno, ${ }^{46}$ che raggiunge i livelli più alti nei gruppi TRA2 e TRA4, seguiti dal gruppo IT2:47

(20) Secondo quanto riportato da un cronista bolognese, Giulio II era determinato a farsi crescere la barba fino a quando *NON AVREBBE SCONFITTO E CACCIATO (CC)/*SCONFIGGEVA E CACCIAVA (IM)/*NON AVRÀ SCONFITTO E CACCIATO (FF) $/{ }^{*}$ NON SCONFIGGEREBBE E CACCEREBBE (C)/*NON SCONFIGGERÀ E CACCERÃ $(\mathrm{F}) /{ }^{\star}$ SCONFIGGE E CACCIA (Pr) dall'Italia il re francese Luigi XII.

\subsubsection{Segnalazione della temporalità relativa nel discorso indiretto libero (DIL)}

Il discorso indiretto libero (DIL) è uno dei discorsi inseriti che si oppongono alla diegesi, cioè al discorso della voce narrante. Il DIL presenta i contenuti da due punti di vista diversi: quello del personaggio, ossia l'enunciatore originario, e quello del narratore (cfr. Miklič 2003b: 94). Di conseguenza assomiglia per certi aspetti al DD e per altri al DI, ma la proporzione dei tratti caratteristici dell'uno o dell'altro dipende dalla sua realizzazione nell'ambito di una determinata lingua. In italiano il DIL prototipico rappresenta le stesse caratteristiche del DD per quanto riguarda la struttura sintattica (frasi indipendenti), le scelte lessicali, la deissi temporale e locale. Assomiglia, invece, al DI per quel che riguarda la deissi personale e l'uso dei paradigmi verbali. In sloveno, d'altro canto, il DIL ha ancora più tratti in comune con il DD, visto che anche i paradigmi verbali rimangono uguali a quelli nel DD (cfr. Miklič 2003b: 95).

Il DIL proposto nel cloze GFC (GFC.9\&11) - si tratta, più esattamente, di un «pensiero indiretto libero» (cfr. Leech/Short 1981: 337) - è interessante soprattutto dal

${ }^{45}$ L'impiego inappropriato del CC: 25\% (IT2), 37,5\% (IT3), 58,33\% (IT4), 32,14\% (TRA2), 62,5\% (TRA3), 33,33\% (TRA4).

46 Mentre gli usi del Pr e del F rientrano nei casi di interferenza totale, l'impiego del FF riflette un'interferenza parziale. Gli studenti che hanno scelto il FF avevano notato il rapporto di anteriorità tra l'azione della dipendente di secondo grado e l'azione della completiva, ma influenzati dal prihodnjik (si bo pustil rasti) non hanno segnalato l'appartenenza dell'intera situazione al passato.

${ }^{47}$ L'interferenza ricopre in media il 16,67\% delle soluzioni nel gruppo IT2, 1'8,33\% nei gruppi IT3 e IT4, il 39,29\% nel gruppo TRA2, il 12,5\% nel gruppo TRA3 e il 22,22\% nel gruppo TRA4. 
punto di vista degli usi dei paradigmi verbali, in quanto non presenta elementi di deissi temporale o locale. Nell'espressione il DIL della versione slovena non differisce dal DD (se non per l'assenza delle virgolette con cui di solito si segnala il DD). Non così nell'originale italiano.

(21) DIL: Ne, še vedno je lepa (SED-ND) - sem si rekel, ko sem jo gledal - morda ni bila še nikoli tako lepa in privlačna (PRET-ND).

(22) DD: »Ne, še vedno je lepa (SED-ND), « sem si rekel, ko sem jo gledal, morda ni bila še nikoli tako lepa in privlačna (PRET-ND)."

(23) DIL: No, era sempre bella (IM) - mi dicevo - contemplandola -, forse non era mai stata cosí bella e attraente (TP).

(24) DD: «No, è sempre bella (Pr) », mi dicevo - contemplandola, «forse non è mai stata così bella e attraente $(\mathbf{P P})$ ».

Con l'inclusione del DIL nel test si è voluta osservare la misura in cui la codificazione diversa del fenomeno in sloveno avrebbe influito sulle soluzioni degli studenti, sia di coloro che avevano già acquisito queste nozioni nel loro percorso universitario (IT4 e TRA4) sia di coloro che ancora non le possedevano. Gli studenti hanno dovuto prima riempire il cloze GFC. In seguito sono stati sottoposti per la seconda volta allo stesso cloze (2GFC), preceduto, però, dalla versione slovena del testo. La versione slovena avrebbe dovuto favorire una comprensione più completa del contenuto, anche se, al contempo, avrebbe potuto provocare l'interferenza.

Nel caso del cloze GFC la combinazione corretta (IM-TP) è stata proposta dal $25,72 \%$ degli studenti e tale percentuale risulta uguale per i due gruppi (IT e TRA). Ho deciso di considerare parzialmente corretta anche la combinazione Pr-PP, tipica del DD. ${ }^{48}$ Tale interpretazione è stata proposta dall' $8,56 \%$ degli studenti, con intensità maggiore tra gli studenti di Italianistica (IT: 14,29\%; TRA: 2,86\%). Il maggior numero di soluzioni inappropriate è stato prodotto dagli studenti di Traduzione (TRA: 71,43\%; IT: 60\%). ${ }^{49}$ La maggior parte delle soluzioni inappropriate riflette l'influsso dello sloveno (40\%). Si tratta di combinazioni IM-IM (27,14\%), Pr-IM (11,43\%) e Pr-TP $(1,43 \%)$. Mentre gli studenti di Traduzione hanno impiegato più volte i due IM (TRA: $34,29 \%$; IT: $20 \%$ ), errore classificato come interferenza parziale, visto che soltanto l'uso del secondo IM è dettato dall'interferenza, gli studenti di Italianistica hanno usato più volte la combinazione Pr-IM (IT: 14,29\%; TRA: 8,57\%), dovuta probabilmente all'interferenza totale. ${ }^{50}$

${ }^{48}$ Ho tenuto conto del fatto che gli studenti avrebbero potuto interpretare il passo come un DD - interpretazione, peraltro, possibile.

49 Percentuali di soluzioni inappropriate in ordine decrescente: $54,55 \%$ (IT3), 58,33\% (IT4, TRA3), $66,67 \%$ (IT2 e TRA3), 85,71\% (TRA2).

50 Percentuali di soluzioni in cui risulta evidente l'influsso dello sloveno: 50\% (TRA2), 45,45\% (IT3), 44,44\% (TRA4), 41,67\% (IT2), 33,33\% (TRA3) e 25\% (IT4). 
La percentuale di scelte appropriate (IM-TP) nella seconda esecuzione del cloze (2GFC) $(15,71 \%)$ è calata rispetto alla percentuale riscontrata nella prima esecuzione (GFC) $(25,72 \%)$. Una quantità minore di soluzioni adeguate è stata proposta dagli studenti di Traduzione (TRA: 11,43\%; IT: 20\%). Ė aumentata la frequenza della combinazione Pr-PP (15,71\%), tranne che nei gruppi IT2 e TRA4, ma raramente a scapito della combinazione IM-TP. ${ }^{51}$ Mentre nel gruppo TRA la quantità di soluzioni inappropriate è ulteriormente cresciuta rispetto al cloze GFC (80\%), nel gruppo IT è rimasta quasi invariata $(57,14 \%) .52$ Tra le soluzioni inappropriate sono aumentati i casi di interferenza (55,71\%), soprattutto i casi di interferenza totale (Pr-IM: 27,14\%) e i casi di interferenza parziale (Pr-TP: 11,43\%). È invece diminuita la quantità dei casi IM-IM (17,14\%) spesso perché sostituita dalla soluzione Pr-IM o, in misura minore, dalla combinazione Pr-PP. ${ }^{53}$ Il confronto tra i gruppi IT e TRA dimostra un aumento maggiore dell'interferenza nel gruppo TRA ( $60 \%$ in $2 \mathrm{GFC}$ vs $42,86 \%$ in GFC) rispetto al gruppo IT ( $51,43 \%$ in 2 GFC vs $37,14 \%$ in GFC). I casi di interferenza sono aumentati in tutti i sottogruppi, tranne che nei gruppi IT4 e TRA3. L'aumento più consistente si è verificato nel gruppo TRA2. 54

Una delle attività successive al cloze 2GFC richiedeva agli studenti di argomentare la propria scelta della forma verbale in 2GFC.9. Il fenomeno è stato riconosciuto come DIL soltanto da 4 studenti del $4^{\circ}$ anno IT, e da uno studente del $4^{\circ}$ anno TRA. ${ }^{55}$ L'uso di IM in 2 GFC.9 è stato per lo più spiegato con il ricorso a concetti quali «durata», «descrizione», «stato», riferiti alla simultaneità nel passato. Chi, invece, aveva proposto il Pr ha motivato la sua scelta con un riferimento al DD. La traduzione slovena come stimolo all'uso del Pr è stata menzionata da 6 studenti di Traduzione. Alcuni hanno scritto di aver scelto il paradigma intuitivamente, ma le loro soluzioni sono state di norma errate.

La soluzione corretta è stata proposta anche da parte di alcuni studenti che non avevano ancora studiato l'uso delle forme verbali nel DIL. Ma in tal caso è stata spesso sostituita, nel cloze $2 \mathrm{GFC}$, da una combinazione caratterizzata dall'interferenza.

\subsubsection{Preludio}

Tra i casi rivelatisi di più difficile soluzione va collocato anche l'impiego del TP per riempire lo spazio vuoto A.1. Nel testo riportato sotto, l'azione erano partiti è in rap-

\footnotetext{
${ }^{51} \mathrm{Si}$ è avuto questo cambiamento in 2 casi su 18. Tre volte la soluzione appropriata è stata sostituita dalla combinazione Pr-TP.

52 Percentuali di soluzioni inappropriate: IT2 (75\%), IT3 (63,64\%), IT4 (33,33\%), TRA2 (92,86\%), TRA 3 (66,67\%), TRA $4(77,78 \%)$.

${ }^{53}$ Le soluzioni con interferenza totale sono aumentate dell' $11,42 \%$ (25,71\%) nel caso del gruppo IT e del $2 \%$ nel caso del gruppo TRA $(28,57 \%)$. Nel gruppo IT sono aumentate del $2,85 \%$ anche le soluzioni con interferenza parziale $(25,71 \%)$, mentre nel gruppo TRA sono, invece, - diminuite (dal $34,29 \%$ al $31,43 \%$ ).

54 Percentuali di soluzioni caratterizzate dall'interferenza: 85,71\% (TRA2), 66,67\% (IT2), 63,64\% (IT3), 55,56\% (TRA4), 33,33\% (TRA3), 25\% (IT4).

55 Tra coloro che hanno riconosciuto il DIL non tutti hanno proposto la combinazione corretta.
} 
porto di contiguità rispetto alle azioni aveva travolti e sepolti. Tutta questa parte iniziale costituisce una specie di preambolo ai fatti nel fuoco narrativo che hanno come centro l'azione (li) hanno raggiunti, espressa per mezzo del PP:

(25) Jean-Daniel e i suoi due bambini ERANO PARTITI (TP) per una gita sulla neve nei dintorni di Villars-sur-Ollon (Svizzera) a bordo della slitta TRAINATA dal loro cane Shady, quando all'improvviso una slavina li AVEVA TRAVOLTI (TP) e SEPOLTI (TP). I soccorritori li hanno raggiunti (PP) solo dopo un certo tempo, ma i tre erano (IM) già fuori pericolo...56

Questo modo di esporre azioni passate in ordine cronologico, usando per quelle all'inizio del testo un paradigma in grado di segnalare l'anteriorità, è denominato preludio: 57 è come se si volesse togliere alle azioni introduttive una parte della loro rilevanza per dare più importanza alle azioni nel fuoco narrativo (cfr. Miklič 1997: 492; Miklič 1998b: 184-185). Visto che tale fenomeno nello sloveno odierno è quasi del tutto sconosciuto (Miklič 1998b: 187) sarebbe stato illusorio aspettarsi dai discenti un uso spontaneo e appropriato del TP. Si è voluto, piuttosto, mettere alla prova la loro capacità di osservazione del cotesto (la presenza dei due TP aveva travolti e sepolti).

Il risultato migliore è stato ottenuto dagli studenti IT (36,11\% vs $20 \%$ di soluzioni corrette e parzialmente corrette), grazie alle soluzioni del $4^{\circ}$ anno IT. ${ }^{58}$ Invece di proporre la soluzione giusta gli studenti hanno fatto uso del PP, scelta condizionata dall'influsso dello sloveno. ${ }^{59}$

\subsubsection{Espressione di notizie riferite ad azioni passate dubbie}

Il condizionale composto (CC) si usa anche per esprimere azioni passate riguardo alla cui verità l'emittente del testo ha delle riserve (Miklič 2003a: 555). In questo modo è espressa, nel cloze $\mathrm{E} / 2 \mathrm{E}$ una riserva riguardo a una delle tesi presentate nel libro Omero nel Baltico, relativa al luogo delle gesta dei Danai:

(26) Secondo quanto afferma l'ingegner Felice Vinci nel libro Omero nel Baltico, le gesta dei Danai, narrate nei poemi omerici, NON SI SAREBBERO SVOLTE (CC) (E.1/2E.1) nel Mediterraneo, ma nei mari del Nordeuropa.

\footnotetext{
${ }^{56}$ Le azioni scritte in lettere maiuscole fanno parte del preambolo, mentre quelle scritte in minuscole sono nel fuoco narrativo.

57 A differenza delle azioni anteriori, che sono verbalizzate all'interno delle dipendenti, le azioni espresse con il TP (o con il PP nel caso del procedimento storico) per segnalare il preludio sono verbalizzate nell'ambito delle frasi indipendenti.

58 Percentuali delle soluzioni errate in ordine decrescente: 91,67\% (IT2), 88,89\% (TRA4), 83,33\% (IT3), 78,57\% (TRA2), 75\% (TRA3), 16,67\% (IT4). Il vantaggio del gruppo IT4 è probabilmente dovuto sia alla conoscenza del fenomeno che alla capacità di osservazione. Il fenomeno era stato studiato anche dagli studenti del $4^{\circ}$ anno TRA e del $3^{\circ}$ anno IT, ma nonostante ciò questi gruppi hanno ottenuto risultati bassi, peggiori di quelli dei gruppi TRA2 e TRA3 che non ne erano a conoscenza.

${ }^{59}$ Uso del PP: 75\% (IT2), 83,33\% (IT3), 8,33\% (IT4), 71,43\% (TRA2), 58,33\% (TRA3), 66,67\% (TRA4).
} 
L'espressione di una tale azione passata non necessariamente richiede l'impiego del CC:

(27) Secondo quanto riportato da un cronista bolognese, Giulio II ERA determinato (IM) (C.10) a farsi crescere la barba fino a quando non avesse sconfitto e cacciato dall'Italia il re francese Luigi XII.

La presenza del sintagma secondo quanto afferma nel cotesto, dunque, potrebbe suggerire al soggetto testato l'impiego del CC. Tuttavia ciò non basta, il che traspare anche dagli elaborati degli studenti. Soltanto il 5,63\%,60 infatti, ne ha fatto uso. Una quantità più elevata ha preferito il $\mathrm{PR}(18,31 \%) .{ }^{61}$

In sloveno l'espressione di notizie riferite ad azioni passate dubbie è affidata al condizionale sloveno preceduto dalla particella naj (naj se ne bi odvijala (POG$\mathrm{ND})$ ), ${ }^{62}$ oppure al preterito sloveno preceduto dalla particella avverbiale baje ((baje) se niso odvijala (PRET-ND)). Anche se nel processo di traduzione da una lingua all'altra, secondo la teoria della traduzione di Coseriu (2002: 385), si tratta di riprodurre la stessa designazione e lo stesso senso ma non gli stessi significati linguistici (strumenti tipici di una determinata lingua), in certi casi è comunque possibile rendere il senso e la designazione nel testo d'arrivo anche per mezzo di un significato analogo a quello presente nel testo di partenza. In questo senso ho voluto verificare in che misura l'impiego del pogojnik nella traduzione ${ }^{63}$ avrebbe influito sulla scelta del mezzo analogo nel cloze $2 \mathrm{E}$ e se gli studenti ne avrebbero riconosciuto l'uso o avrebbero agito a seconda dell'analogia formale.

(28) Po trditvah inženirja Feliceja Vincija v delu Homer na Baltiku naj BI SE junaška dela Danajcev, o katerih pripovedujejo Homerjeve pesnitve, NE ODVIJALA (POGND) v Sredozemlju, pač pa na morjih Severne Evrope.

Rispetto a E.1 il numero di occorrenze del CC in E.2 è aumentato (19,72\%).64 L'impiego è stato maggiore nell'ambito del gruppo TRA (TRA: 25,71\%; IT: 13,89\%). È aumentata anche la quantità di usi del PR (21,13\%). ${ }^{65}$ Alcuni studenti hanno detto di aver scelto il CC per aver notato il pogojnik nella traduzione. Nonostante questa affermazione è difficile stabilire con certezza se la scelta sia stata motivata dalla forma o dalla consapevolezza dell'uso. Visto che non è stato usato il C, che avrebbe rappresentato il valore prototipico più simile al pogojnik, si potrebbe dire che agli stu-

\footnotetext{
${ }^{60} \mathrm{Si}$ tratta di 3 studenti del gruppo IT e di uno studente del gruppo TRA.

61 Percentuali delle soluzioni con il PR: 25\% (IT2), 16,67\% (IT3), 8,33\% (IT4), 14,29\% (TRA2), $33,33 \%$ (TRA3), $11,11 \%$ (TRA4).

62 Sarebbe possibile esprimerla anche per mezzo della combinazione naj e condizionale passato sloveno (naj bi se ne bila odvijala).

$63 \mathrm{Il}$ cloze $2 \mathrm{E}$ è stato preceduto dalla traduzione del testo integrale in sloveno.

64 Usi del CC: 8,33\% (IT2), 16,67\% (IT3, IT4), 14,29\% (TRA2), 33,33\% (TRA3, TRA4).

65 Gli usi del PR: 33,33\% (IT2), 16,67\% (IT3, IT4), 7,14\% (TRA2), 33,33\% (TRA3), 22,22\% TRA4.
} 
denti è mancata soprattutto la terminologia con cui spiegare correttamente il fenomeno. Altri hanno attribuito la scelta del $\mathrm{CC}$ a concetti quali «espressione di una supposizione», «espressione del sentito dire». Solo uno studente del gruppo IT3 ha spiegato la propria scelta usando la terminologia impiegata in classe: «l'espressione di un'azione riguardo alla quale il parlante ha delle riserve».

La quantità di soluzioni inappropriate in E.1 è del 74,65\% (è maggiore nel gruppo TRA (TRA: 77,14\%; IT: 72,22\%)), ${ }^{66}$ mentre nel caso di 2E.1 risulta più bassa $(59,15 \%)$ (stavolta soprattutto nel gruppo TRA (TRA: 54,29\%; IT: 63,89\%)). ${ }^{67}$

Tra le soluzioni inappropriate spiccano quelle scelte per l'interferenza: il PP e l'IM. Mentre la scelta del PP è stata determinata, probabilmente, dalla consapevolezza degli studenti di avere di fronte un fatto passato, la scelta dell'IM rappresenta un grado di interferenza maggiore: riflette, infatti, l'influsso del nedovršnik nelle due forme verbali (se niso odvijala (PRET-ND); bi se ne odvijala (POG-ND). In E.1, la quantità di PP $(23,94 \%)$ è quasi uguale alla quantità di IM $(21,13 \%)$. In entrambi i casi la percentuale è un po' più alta nell'ambito del gruppo IT (PP: 27,78\%, IM: $22,22 \%$ vs PP: $20 \%$, IM: $20 \%){ }^{68}$ In 2 E. 1 si nota un impiego minore dell'IM $(19,71 \%)$ e del PP $(15,49 \%)$ rispetto a E.1. La quantità di usi nell'ambito dei due gruppi è più o meno uguale (IM: 19,44\% (IT), 20\% (TRA); PP: 16,67\% (IT), 14,29\% (TRA)). ${ }^{69}$

\subsubsection{Scelta tra la narrazione e la descrizione}

Il sistema aspettuale italiano e quello sloveno funzionano in modo diverso. Esistono, tuttavia, delle situazioni in cui si può riscontrare una corrispondenza, sebbene superficiale, nella distribuzione dei perfetti (P) e degli imperfetti (IM) da una parte e dei verbi perfettivi (dovršnik (D)) e imperfettivi sloveni (nedovršnik (ND)) dall'altra:

(29) Ko sem vstopil (PRET-D), je brala (PRET-ND).

(30) Quando sono entrato (PP)/entrai (PR) stava leggendo (IM).

Avendo notato tale corrispondenza il discente è portato a scegliere i P e gli IM a seconda dei modelli della madrelingua (Miklič 1983: 59).

Uno degli ambiti in cui la scelta tra il P e l'IM da un lato e tra il D e il ND dall'altro segue un andamento diverso è rappresentato dalla coordinazione copulativa (e dall'asindeto): mentre la lingua slovena può presentare uno accanto all'altro un D e un ND, scelti in base alla natura di ogni singola azione (telica o atelica), l'italiano mostra la tendenza a mantenere l'ottica costante (narrazione (con i P) o descrizione (con gli IM)) (Miklič 1983: 111).

66 E.1: 66,67\% (IT2), 75\% (IT3 e IT4), 85,71\% (TRA2), 58,33\% (TRA3), 88,89\% (TRA4).

67 2E.1: 58,33\% (IT2), 66,67\% (IT3 e IT4), 78,57\% (TRA2), 33,33\% (TRA3), 44,44\% (TRA4).

68 L'uso del PP (E.1): 33,33\% (IT2), 25\% (IT3, IT4), 21,43\% (TRA2), 16,67\% (TRA3), 22,22\% (TRA4). L'uso dell'IM: 8,33\% (IT2), 33,33\% (IT3), 25\% (IT4), 28,57\% (TRA2), 8,33\% (TRA3), 22,22\% (TRA4).

${ }^{69}$ L'uso del PP (2E.2): 16,67\% (IT2), 25\% (IT3), 8,33\% (IT4), 21,43\% (TRA2), 8,33\% (TRA3), 11,11\% (TRA4). L'uso dell'IM: 8,33\% (IT2), 25\% (IT3, IT4), 35,71\% (TRA2), 8,33\% (TRA3), 11,11\% (TRA4). 
In C.3-5 nella versione slovena stanno due ND (so pisali, so primerjali), ma l'azione in C.3 potrebbe essere resa anche per mezzo di un D (so zapisali). Nel testo italiano, invece, le due azioni possono essere presentate sia entrambe globalmente per mezzo dei perfetti (narrazione) sia entrambe corsivamente (v. sopra) per mezzo degli imperfetti (descrizione). Con la scelta dei due perfetti si continua la serie di azioni centrali iniziata con incominciò a sfoggiare, con la scelta dei due imperfetti, invece, si passa subito alle azioni che fanno da sfondo all'azione centrale. La considerazione dell'intero testo e del suo carattere informativo suggerisce l'uso dei due P. Inadeguata è invece la combinazione P-IM.

(31) Proti koncu leta 1510 je papež Julij II začel razkazovati (PRET-D + NED-ND ${ }^{70}$ ) svojo gosto brado, nad katero so se zgražali (PRET-ND) kardinali in poslaniki. Nekateri SO PISALI (PRET-ND)/SO ZAPISALI (PRET-D), da papež spominja (SED-ND) na medveda, drugi $\underline{\text { SO }}$ ga PRIMERJALI (PRET-ND) z eremiti.

(32) Verso la fine del 1510, papa Giulio II incominciò a sfoggiare (PR+INF) una folta barba, che scandalizzava (IM) cardinali e ambasciatori: alcuni SCRISSERO (PR) che il pontefice somigliava (IM) a un orso, altri lo PARAGONARONO (PR) invece a un eremita.

Soluzione alternativa: SCRIVEVANO-PARAGONAVANO (IM-IM).

Soluzioni inappropriate: *scrissero-paragonavano (PR-IM), *hanno scritto-paragonavano (PP-IM).

La combinazione con due PR è stata proposta dal 18,31\% degli studenti (IT: 19,44\%; TRA: $17,14 \%$ ), quella con due IM, invece, dal 45,07\% (IT: 47,22\%; TRA: 42,86\%). D'altro lato il 39,39\% degli studenti (IT: 30,56\%; TRA: 34,29\%) non ha mantenuto l'ottica costante, proponendo, quindi, combinazioni PP-IM oppure PR-IM.

Per il parlante sloveno, la scelta tra il perfetto e l'imperfetto si rivela particolarmente difficile quando, soprattutto nell'ambito di una indipendente, si trova davanti a un'azione (o più) della storia focale, di carattere atelico e, quindi, espressa per mezzo del ND, visto che in italiano tale azione - in linea di massima - potrebbe essere colta globalmente e, quindi, resa con il $\mathrm{P}$, oppure vista corsivamente (v. sopra) e, quindi, denotata per mezzo dell'IM (cfr. Miklič 1981: 81). È lui stesso a dover decidere - valutando gli elementi di co- e contesto - se presentarla con il P o con l'IM. Gli studenti si sono trovati davanti a questo tipo di scelta nei punti A.3, C.2, C.6, C.10, E.5/2E.5, GFC.4/2GFC.4. In tutti questi casi i testi italiani prevedevano l'impiego dell'IM, uso che per l'analogia con lo sloveno (l'uso dei ND) non avrebbe dovuto presentare grandi difficoltà. I risultati dell'analisi hanno, però, rivelato che una determinata azione viene percepita come un'azione da sfondo (IM) con più facilità in certi contesti che non in altri. In C.10, ad es., gli studenti che hanno presentato l'azione essere determinato con il $\mathrm{P}$ ( $f u$ determinato, è stato determinato) (49,3\%; IT: 41,67\%; TRA: $57,14 \%$ ) sono stati più numerosi di quelli che l'hanno resa con l'IM (45,07\%; IT: 47,22\%, TRA: 42,86\%). In C.6 la situazione si è rivelata simile:

70 NED-ND: forma imperfettiva dell'infinito sloveno. 
(33) Verso la fine del 1510, papa Giulio II incominciò a sfoggiare una folta barba che scandalizzava cardinali e ambasciatori: alcuni scrissero che il pontefice somigliava a un orso, altri lo paragonarono invece a un eremita. In realtà, con tale gesto il Papa INTENDEVA (IM) C.6 emulare Giulio Cesare, il quale nel 54 a.C. aveva giurato di non radersi finché avesse vendicato la morte dei propri uomini massacrati dai Galli. Secondo quanto riportato da un cronista bolognese, Giulio II ERA DETERMINATO (IM) C.10 a farsi crescere la barba fino a quando avesse sconfitto e cacciato dall'Italia il re francese Luigi XII. (LSE 3993-4)

In GFC.4 (similmente in 2GFC.4) - trattandosi di un caso di descrizione prototipico, segnalato già dall'impiego di ne rimaneva nel cotesto vicino - l'IM è stato scelto da quasi tutti gli studenti (92,86\%; IT: 91,43\%; TRA: 94,29\%):

(34) Mi accostai dunque al letto, dalla parte dei piedi, restando lí, con le mani appoggiate alla ringhiera. Benché infilata sotto le coperte, Micòl ne rimaneva fuori con tutto il busto. INDOSSAVA (IM) un pullover verde-scuro, accollato e dalle maniche lunghe, la medaglietta d'oro dello sciaddài scintillante sopra la lana della maglia, e due guanciali a sostegno della schiena.

L'impiego del PR in C.2, rappresenta, invece, un'interpretazione accettabile:

(35) Verso la fine del 1510, papa Giulio II incominciò a sfoggiare una folta barba SCANDALIZZÒ (PR) cardinali e ambasciatori.

In questo caso 1'IM è stato proposto dal 38,03\% (IT: 38,89\%; TRA: 37,78\%) degli studenti, il PR dal 43,66\% (IT: 38,89\%; TRA: 48,57\%).

L'impiego generalizzato del PR in alcuni contesti, tuttavia, non è un cattivo segno: vuol dire che gli studenti cercano di non prendere come modello la codificazione slovena, e di partire, invece, dalla situazione italiana.

Le difficoltà relative alla scelta tra il $\mathrm{P}$ e l'IM si sono potute notare anche in GFC.3/2GFC.3. Il predicato $f u$ rappresenta assieme al predicato persuase un'unica azione telica, colta globalmente. Nell'espressione di azioni teliche in sloveno viene di norma accentuata la fase puntuale tramite l'uso del D. Il verbo essere sloveno, però, dispone soltanto della forma imperfettiva (je bil, ND). Sotto l'influsso dell'utilizzo del ND sloveno la maggioranza degli studenti ha riempito lo spazio vuoto con 1'IM (GFC.3: 52,86\%; 48,57\% (IT); 57,14\% (TRA); 2GFC.3: 50\%; 45,71\% (IT); $54,29 \%$ (TRA)).

(36) Mi bastò entrare nella sua stanza, invece, (dopo avermi introdotto, Perotti aveva richiuso discretamente la porta dietro le mie spalle), per vedere che mi sorrideva benigna, gentile, amica. Ancor più dell'esplicito invito a venire avanti, FU (PR) quel suo sorriso luminoso, pieno di tenerezza e di perdono, che mi persuase (PR) a staccarmi dal fondo buio della stanza e ad avvicinarmi.

Soluzione inappropriata più frequente: *ERA (IM). 


\subsubsection{Segnalazione dell'appartenenza delle azioni di un testo a mondi testuali separati}

Le azioni presentate nel testo E (Danai) appartengono a due diversi mondi testuali. Il primo, staccato dal momento dell'enunciazione, ha come protagonista il popolo dei Danai, ragione per cui viene presentato per mezzo del passato remoto (E.7) o dei suoi sostituti - condizionale composto per sottolineare la sfumatura modale di notizia riferita in E.1 e participio passato nel caso della dipendente implicita in E.6. Il secondo mondo testuale, strutturato attorno alle tesi dell'autore del libro e legato al momento attuale, richiede, invece, l'impiego del passato prossimo (E.3):

(37) Secondo quanto afferma l'ingegner Felice Vinci nel libro Omero nel Baltico, le gesta dei Danai, narrate nei poemi omerici, NON SI SAREBBERO SVOLTE (CC) E.1 nel Mediterraneo, ma nei mari del Nordeuropa. Partendo da uno scritto di Plutarco, nel quale lo storico greco sostiene che l'isola di Calipso, Ogigia, è situata a cinque giorni di navigazione dalla Britannia, Vinci HA ANALIZZATO (PP) E.3 numerosi toponimi dell'Europa settentrionale, sorprendentemente simili a quelli omerici, nonché svariati fenomeni naturali, come gli enormi gorghi d'acqua detti maelstrom, che ricordano Scilla e Cariddi. Egli Ė quindi GIUNTO a sostenere che i Danai (nome con cui Omero indicava i Greci) ERANO in realtà Danesi dell'età del bronzo, MIGRATI (PART) E.6 in seguito nel Mediterraneo, dove ATTRIBUIRONO (PR) E.7 alle nuove terre i toponimi dei luoghi natii. (LSE 3976-12)

In sloveno le azioni passate di entrambi i mondi testuali - eccetto l'azione non si sarebbero svolte - sarebbero espresse per mezzo del preteklik. Gli studenti, poco abituati alle situazioni in cui all'interno di uno stesso testo per le azioni della diegesi compaiono sia il PP che il PR, ${ }^{71}$ hanno avuto difficoltà ad usare correttamente i due paradigmi: solo 4 studenti di Italianistica e 2 studenti di Traduzione hanno usato una delle due combinazioni accettabili (CC-PP-PR; PR-PP-PR) per riempire gli spazi vuoti E.1-E.3-E.7.

${ }^{71}$ Durante la normale prassi didattica gli studenti sono generalmente sottoposti ai cloze che prevedono o la scelta del PR o la scelta del PP. Sono quindi escluse le situazioni che prevedono la coesistenza dei due paradigmi. 


\subsection{Osservazioni generali e confronto tra i gruppi}

\begin{tabular}{|l|c|c|c|c|c|c|c|c|c|c|c|c|}
\hline GRUPPO & \multicolumn{3}{|c|}{ IT 2 } & \multicolumn{3}{c|}{ IT3 } & \multicolumn{3}{c|}{ IT4 } & \multicolumn{3}{c|}{ IT } \\
\hline$\%$ & corr. & pcorr. & err. & corr. & pcorr. & err. & corr. & pcorr. & err. & corr. & pcorr. & err. \\
\hline ESERCIZIO A & 29,17 & 18,75 & 52,08 & 47,92 & 14,58 & 37,5 & 85,42 & 8,33 & 6,25 & 54,17 & 13,89 & 31,94 \\
\hline ESERCIZIO B & 36,67 & 6,67 & 56,67 & 53,33 & 3,33 & 43,33 & 66,67 & 5 & 28,33 & 52,22 & 5 & 42,78 \\
\hline ESERCIZIO C & 55 & 8,33 & 36,67 & 59,17 & 4,17 & 36,67 & 61,67 & 0,83 & 37,5 & 58,61 & 4,44 & 36,94 \\
\hline ESERCIZIO D1 & 44,44 & 4,17 & 51,39 & 47,22 & 12,5 & 40,28 & 56,94 & 16,67 & 26,39 & 49,54 & 11,11 & 39,35 \\
\hline ESERCIZIO D2 & 51,39 & 2,78 & 45,83 & 45,83 & 11,11 & 43,06 & 55,56 & 18,06 & 26,39 & 50,93 & 10,65 & 38,43 \\
\hline ESERCIZIO E1 & 40,48 & 9,52 & 50 & 48,81 & 3,57 & 47,62 & 55,95 & 2,38 & 41,67 & 48,41 & 5,16 & 46,43 \\
\hline ESERCIZIO E2 & 47,62 & 8,33 & 44,05 & 51,19 & 3,57 & 45,24 & 60,71 & 3,57 & 35,71 & 53,17 & 5,16 & 41,67 \\
\hline ESERCIZIO GFC1 & 33,33 & 7,5 & 59,17 & 58,18 & 5,45 & 36,36 & 56,67 & 1,67 & 41,67 & 49,14 & 4,86 & 46 \\
\hline ESERCIZIO GFC2 & 40,83 & 8,33 & 50,83 & 58,18 & 4,55 & 37,27 & 60,83 & 5,83 & 33,33 & 53,14 & 6,29 & 40,57 \\
\hline EESERCIZI & 42,10 & 8,26 & 49,63 & 52,20 & 6,98 & 40,81 & 62,27 & 6,93 & 30,80 & 52,15 & 7,49 & 40,46 \\
\hline
\end{tabular}

Tabella 3: Percentuali relative alle soluzioni corrette, parzialmente corrette e inappropriate, per ciascun esercizio e per l'insieme degli esercizi, proposte dagli studenti di Italianistica

\begin{tabular}{|l|l|l|l|l|l|l|l|l|l|l|l|l|}
\hline GRUPPO & \multicolumn{3}{|c|}{ TRA2 } & \multicolumn{3}{c|}{ TRA3 } & \multicolumn{3}{c|}{ TRA4 } & \multicolumn{3}{c|}{ TRA } \\
\hline$\%$ & corr. & pcorr. & err. & corr. & pcorr. & err. & corr. & pcorr. & err. & corr. & pcorr. & err. \\
\hline ESERCIZIO A & 50 & 10,71 & 39,29 & 60,42 & 6,25 & 33,33 & 55,56 & 13,89 & 30,56 & 55 & 10 & 35 \\
\hline ESERCIZIO B & 54,29 & 5,71 & 40 & 60 & 0 & 40 & 55,56 & 0 & 44,44 & 56,57 & 2,29 & 41,14 \\
\hline ESERCIZIO C & 52,86 & 2,86 & 44,29 & 61,67 & 0 & 38,33 & 56,67 & 2,22 & 41,11 & 56,86 & 1,71 & 41,43 \\
\hline ESERCIZIO D1 & 51,19 & 16,67 & 32,14 & 59,72 & 8,33 & 31,94 & 48,15 & 7,41 & 44,44 & 53,33 & 11,43 & 35,24 \\
\hline ESERCIZIO D2 & 48,81 & 16,67 & 34,52 & 62,5 & 6,94 & 30,56 & 51,85 & 5,56 & 42,59 & 54,29 & 10,48 & 35,24 \\
\hline ESERCIZIO E & 41,84 & 3,06 & 55,10 & 46,43 & 7,14 & 46,43 & 50,79 & 6,35 & 42,86 & 45,71 & 5,31 & 48,98 \\
\hline ESERCIZIO E2 & 50 & 3,06 & 46,94 & 57,14 & 9,52 & 33,33 & 55,56 & 11,11 & 33,33 & 53,88 & 7,35 & 38,78 \\
\hline ESERCIZIO GFC1 & 52,86 & 2,14 & 45 & 55 & 2,5 & 42,5 & 47,78 & 4,44 & 47,78 & 52,28 & 2,86 & 44,86 \\
\hline ESERCIZIO GFC2 & 47,86 & 2,85 & 49,29 & 56,67 & 5 & 38,33 & 54,44 & 4,44 & 41,11 & 52,57 & 4 & 43,43 \\
\hline EESERCIZI & 49,97 & 7,08 & 42,95 & 57,73 & 5,08 & 37,19 & 52,93 & 6,15 & 40,91 & 53,39 & 6,16 & 40,46 \\
\hline
\end{tabular}

Tabella 4: Percentuali relative alle soluzioni corrette, parzialmente corrette e inappropriate, per ciascun esercizio e per l'insieme degli esercizi, proposte dagli studenti di Traduzione

Le due tabelle rappresentano il quadro riassuntivo della prestazione linguistica di ciascun gruppo. In generale i dati rivelano una modesta prevalenza delle soluzioni appropriate $(52,77 \%)$ e parzialmente corrette $(6,83 \%)$ rispetto a quelle inappropriate $(40,46 \%)$. Le soluzioni appropriate in assoluto rivelano un modesto vantaggio degli studenti di Traduzione (TRA: 53,39\%; IT: 52,15\%). In 5 cloze su 9 gli studenti di Traduzione e di Mediazione hanno ottenuto un punteggio più alto rispetto agli studenti di Italianistica. Tuttavia, se consideriamo separatamente i singoli sottogruppi, notiamo che il risultato migliore è stato ottenuto dagli studenti del $4^{\circ}$ anno di Italianistica $(69,2 \%)$, seguiti dal $3^{\circ}$ anno di Mediazione $(62,81 \%)$. Il 
terzo posto è stato raggiunto dal $3^{\circ}$ anno di Italianistica $(59,18 \%)$ che precede di pochissimo il $4^{\circ}$ anno di Traduzione $(59,08 \%)$ e il $2^{\circ}$ anno di Mediazione (57,05\%). All'ultimo posto, infine, si è classificato il $2^{\circ}$ anno di Italianistica $(50,36 \%) .{ }^{72}$ In linea di massima la classifica riassuntiva conferma, fatta eccezione per il quarto anno di Traduzione, un miglioramento della prestazione legato al grado di avanzamento nel percorso degli studi universitari.

Se consideriamo l'esito di ogni singolo esercizio, la correlazione positiva tra anno di corso frequentato e prestazione grammatico-testuale si nota sei volte negli studenti di Italianistica e tre volte in quelli di Traduzione; nei casi rimanenti siamo in presenza di un'oscillazione, ossia di assenza di correlazione tra miglioramento della prestazione e anno di corso frequentato. Nei casi di oscillazione il gruppo IT4 si classifica una volta all'ultimo posto, mentre il gruppo TRA4 ottiene il risultato peggiore quattro volte. L'esame più dettagliato delle soluzioni con cui è stato riempito ogni singolo spazio vuoto mostra - negli studenti di Italianistica - un miglioramento delle capacità grammaticotestuali in dipendenza dagli anni di studio in 37 casi, un peggioramento in 5 casi, un'oscillazione in 22 casi $^{73}$ e un caso di prestazione costante. La situazione concernente gli studenti di Mediazione e di Traduzione vede, invece, 11 casi di miglioramento, 44 casi di oscillazione, ${ }^{74} 9$ casi di peggioramento e un caso di prestazione costante.

La situazione vede più volte il verificarsi di un progresso legato all'anno di corso frequentato nell'ambito del gruppo di Italianistica, ${ }^{75}$ mentre il gruppo di Traduzione e Mediazione si dimostra più omogeneo e forse meno incline a migliorare i risultati con il passare degli anni di studio, il che si potrebbe considerare, in misura limitata, una comprova del fatto che meno insegnamento esplicito e osservazione intenzionale del funzionamento del sistema verbale nel testo e più lavoro su base testuale non significherebbero necessariamente un vantaggio per quel che riguarda l'uso dei paradigmi verba1i. ${ }^{76}$ D'altro lato il buon risultato ottenuto soprattutto dall'IT4 potrebbe dare conferma, appunto, dell'influenza positiva del maggior numero di ore dedicate all'insegnamento esplicito, anche se non si dovrebbe prescindere del tutto dall'importanza delle preconoscenze. I due gruppi migliori, IT4 e TRA3, si inseriscono, infatti, anche tra i tre gruppi migliori per il livello di preconoscenze. Senonché gli studenti del gruppo TRA2, bravi per le preconoscenze, si classificano soltanto al penultimo posto per quanto riguarda l'appropriatezza nell'uso dei paradigmi verbali. Tuttavia il confronto fra i gruppi TRA2 e IT2 di nuovo si rivela a favore del gruppo TRA2, caratterizzato da un livello più alto di preconoscenze. Considerati i risultati in generale, la prestazione degli studenti (solo due gruppi raggiungono una percentuale relativa agli usi appropriati superiore al 60\%) si rive-

${ }^{72}$ Sono state prese in considerazione le medie delle percentuali indicanti gli usi corretti e parzialmente corretti delle forme verbali.

73 In 5 su 22 casi di oscillazione il gruppo IT4 si classifica all'ultimo posto, in 6, invece, al primo.

74 In 17 su 44 casi di oscillazione il gruppo TRA4 si classifica all'ultimo posto, in 8, invece, al primo.

75 Tale conclusione è abbastanza logica, visto che i gruppi IT4 e IT2 rappresentano i due estremi della classifica.

76 Per poter essere del tutto sicuri di questa affermazione bisognerebbe eseguire un esperimento del genere con ciascun gruppo più volte nell'arco degli anni di studio. 
la deludente. ${ }^{77}$ Sembra che, nonostante l'insegnamento esplicito, l'impiego delle forme verbali permanga un campo difficile da maneggiare con disinvoltura.

I risultati delle analisi rivelano, inoltre, che il problema principale degli studenti consiste non nella difficoltà di comprendere le caratteristiche del mondo testuale, ma nella loro interpretazione consapevole, che dovrebbe portare all'uso corretto delle forme verbali. Visto che la capacità di valutare in modo cosciente il mondo testuale viene acquisita nell'ambito dell'insegnamento degli usi, si potrebbe dire che gli studenti possiedono un sapere non abbastanza consolidato, oppure che trovano difficoltà nell'applicare gli usi alla realtà extralinguistica per poca esperienza in merito al funzionamento dei paradigmi all'interno di vari tipi di testo. Potrebbero essere spia di un sapere non abbastanza consolidato - oltre ai numerosi casi di interferenza - anche l'impiego generalizzato del trapassato remoto, ${ }^{78}$ l'uso mancato, oppure generalizzato del congiuntivo ${ }^{79}$ ecc., mentre si potrebbero ascrivere alle difficoltà nell'applicare gli usi alla realtà extralinguistica l'uso generalizzato del passato prossimo nei contesti in cui andrebbe usato il passato remoto, tipico soprattutto degli studenti del $2^{\circ}$ anno di Italianistica, la distinzione tra la narrazione e la descrizione, nonché, nel caso di preludio, la decisione di dare rilevanza alle azioni focali con l'impiego cataforico del trapassato prossimo per denotare quelle iniziali. La tesi viene confermata anche dalle difficoltà incontrate dagli studenti qualora si trovino nella necessità di usare, all'interno di uno stesso testo, passato prossimo e passato remoto (si veda il caso del testo $\mathrm{E} / 2 \mathrm{E}$ ).

\section{CONCLUSIONE}

I risultati della ricerca sulle scelte delle forme verbali italiane nei cloze degli studenti dei corsi di laurea in Lingua e letteratura italiana da una parte, e in Traduzione e Mediazione interlinguistica dall'altra hanno confermato la necessità e l'importanza dell'insegnamento e dell'osservazione espliciti del funzionamento del sistema verbale italiano. Infatti, persino a livello universitario, spesso dopo non pochi anni di contatto con la lingua italiana, si è rivelata forte, specialmente in alcuni contesti, l'interferenza, dovuta soprattutto alla codificazione diversa della temporalità e dell'aspettualità nella

$77 \mathrm{Si}$ deve comunque considerare il fatto che il test non era stato annunciato in anticipo.

78 Gli studenti si servono del TR per denotare un'azione anteriore nel passato anche al di fuori di una dipendente temporale: In realtà con tale gesto il Papa intendeva emulare Giulio Cesare, il quale nel 54 a.C. * EBBE GIURATO di non radersi... L'impiego generalizzato del TR si può notare sporadicamente in tutti i gruppi, con intensità maggiore nel gruppo IT4.

7914 studenti, tra cui 6 del $3^{\circ}$ anno di Italianistica, hanno scelto l'imperfetto del congiuntivo in C. 4 perché pensavano che si trattasse dell'espressione di un'opinione di cardinali e ambasciatori: alcuni scrissero che il pontefice *SOMIGLIASSE a un orso, altri lo paragonarono invece a un eremita... Si tratta, invece, dell'atto di riferire la loro opinione e quindi di un fatto reale. Un altro caso di generalizzazione riguarda l'esempio D.1: Il biblico patriarca Giacobbe decise che al suo ultimo figlio ${ }^{*}$ DESSE il nome augurale di Ben Yamin... Per poca esperienza gli studenti non si sono resi conto del fatto che l'uso del congiuntivo sarebbe stato appropriato solo se i soggetti della principale e della dipendente fossero stati diversi. Dato che i soggetti sono uguali non si tratta di una volizione, ma piuttosto di una decisione che riguarda un fatto posteriore nel passato. Il paradigma da usare è quindi il CC (avrebbe dato). È da notare anche l'uso sporadico del congiuntivo nelle frasi indipendenti per l'espressione di un parere o di un dubbio: No, era sempre bella - mi dicevo - contemplandola -, forse non *FOSSE STATA mai cosí bella e attraente. 
prima lingua dei discenti. Hanno riempito i cloze con maggiore successo gli studenti del $4^{\circ}$ anno di Italianistica, seguiti, nell'ordine, da quelli del $3^{\circ}$ anno di Mediazione, del $3^{\circ}$ anno di Italianistica, de $4^{\circ}$ anno di Traduzione, del $2^{\circ}$ anno di Mediazione e, infine, del $2^{\circ}$ anno di Italianistica. In base a questa graduatoria si può supporre che l'insegnamento esplicito della grammatica abbia un influsso decisamente positivo sulla prestazione linguistica; d'altro lato però, si è rivelato importante anche il livello delle preconoscenze.

Le analisi hanno, inoltre, dimostrato che le scelte inappropriate sono legate non tanto alle difficoltà nella ricostruzione del mondo testuale quanto a una mancata interpretazione consapevole delle relazioni tra le azioni che ne fanno parte, e, quindi, all'assenza di un uso consapevole e argomentato delle forme verbali. Alcune volte tale sapere pare non abbastanza consolidato, altre volte le difficoltà sono causate dall'incapacità di applicare il proprio sapere a una concreta attività testuale. Per superare le difficoltà si dovrebbero potenziare ulteriormente le abilità degli studenti nell'ambito della rappresentazione del mondo testuale: partendo dalla loro capacità di recepire in modo abbastanza completo il contenuto di un testo si potrebbe cercare di sviluppare una sensibilità maggiore verso la stratificazione temporale, modale e aspettuale dei testi. Per facilitare l'acquisizione di queste abilità si potrebbero offrire agli studenti, soprattutto ai livelli iniziali, testi narrativi brevi ma completi. Occorrerebbe, in seguito, più lavoro pratico con diversi tipi di testo per evidenziare come la scelta delle forme verbali sia in stretta correlazione anche con la specificità delle diverse tipologie testuali. Gli studenti dovrebbero diventare più abili nella scelta tra il passato remoto e il passato prossimo per rappresentare le azioni centrali di un dato testo. Dovrebbero essere sottoposti anche a testi in cui, a causa dell'appartenenza delle azioni a mondi testuali distinti, vengono usati sia il passato prossimo che il passato remoto. Siccome l'influsso della prima lingua è un fatto da tenere presente nell'apprendimento della lingua in questione, sarebbero da proporre delle attività basate sul principio contrastivo. Si potrebbe partire, ad esempio, dall'analisi attenta del testo sloveno con l'intento di osservare i fenomeni testuali e i rispettivi mezzi linguistici per poi procedere con la traduzione in italiano, concentrandosi soprattutto sulla codificazione appropriata di detti fenomeni. Infine, si potrebbero individuare i punti critici. L'insegnante dovrebbe cercare di richiamare l'attenzione sui principi dell'espressione della stessa realtà anche nell'ambito di qualche altra lingua straniera nota agli studenti, come l'inglese, per aiutarli a raggiungere un livello più alto di comprensione dei rapporti interlinguistici.

\section{Corpus}

LSE = La settimana enigmistica 3976.

LSE = La settimana enigmistica 3993.

GFC = BASSANI, Giorgio (1962) Il giardino dei Finzi-Contini. Torino: Einaudi.

\section{Bibliografia}

BertinetTo, Pier Marco (2003) Tempi verbali e narrativa italiana dell'Otto/Novecento: quattro esercizi di stilistica della lingua. Alessandria: Edizioni dell'Orso.

Bertinetto, Pier Marco/Mario SQuartini (1996) «La distribuzione del Perfetto Semplice e del Perfetto Composto nelle diverse varietà di italiano.» Romance Philology XLIX/4, 384-419. 
COSERIU, Eugenio (2002 [1978]) «Napačna in pravilna izhodišča v teoriji prevajanja.» Trad. Martina Ožbot. In: M. Ožbot (a cura di), Prevajanje srednjeveških in renesančnih besedil: 27. prevajalski zbornik. Ljubljana: Društvo slovenskih književnih prevajalcev, 380-397. [«Falsche und richtige Fragestellungen in der Übersetzungstheorie.» In: L. Grähs/G. Korlén/B. Malmberg (a cura di) Theory and Practice of Translation. (Nobel Symposium 39, Stockholm, Sept. 6-10.). Bern, Frankfurt/Main, Las Vegas: Lang, 17-32.]

LEECH, Geoffrey N./Michael SHORT (1981) Style in fiction (A linguistic introduction to English fictional prose). London e New York: Longman.

MertelJ, Darja (2008) «Ravni znanja večstavčne skladnje pri učencih z italijanščino kot drugim/tujim jezikom.» In: J. Skela (a cura di), 348-369.

MIKLIČ, Tjaša (1981) Kriteriji izbire med perfektom in imperfektom v primerjavi s kriteriji izbire med dovršniki in nedovršniki: Italijansko-slovenska kontrastivna analiza. Ljubljana: Filozofska fakulteta.

MikLIČ, Tjaša (1983) «L'opposizione italiana perfetto vs imperfetto e l'opposizione slovena dovršnost vs nedovršnost nella verbalizzazione delle azioni passate.» Linguistica 23, 53-123.

MikLIČ, Tjaša (1991) «Forme verbali italiane: come vengono presentate dalle grammatiche e come funzionano nei testi.» Scuola Nostra 23, 87-103.

Miklı̌, Tjaša (1992a) «La Consecutio Temporum in sloveno e in italiano: alcune osservazioni.» In: A. G. Mocciaro/G. Soravia (a cura di), L'Europa linguistica: contatti, contrasti, affinità di lingue: atti del XXI Congresso internazionale di studi della Società di linguistica italiana, Catania, 10 -12 settembre 1987. Roma: Bulzoni, 189-217.

MikLIČ, Tjaša (1992b) «Tendenze nella scelta delle forme verbali italiane in testi prodotti da apprendenti sloveni: in cerca di spie dell'interferenza.» In: B. Moretti/D. Petrini/S. Bianconi (a cura di), Linee di tendenza dell'italiano contemporaneo. Roma: Bulzoni, 475-492.

MikLIČ, Tjaša (1997) «Segnalazione della temporalità nel testo: che cosa aiuta il ricevente a collocare le azioni sull'asse temporale.» In: L. Agostiniani et al. (a cura di), Atti del Terzo convegno della Società internazionale di linguistica e filologia italiana. Vol. II. Napoli: Scientifiche italiane, 477-505.

MikLIČ, Tjaša (1998a) «La consecutio temporum italiana nelle vignette: 1'uso delle forme verbali nei testi combinati figurativo-verbali della rivista La settimana enigmistica.» In: M. T. Navarro Salazar (a cura di), Italica Matritensia - Atti del IV convegno SILFI. Firenze: Cesati, 371-382.

MikLIČ, Tjaša (1998b) «Uso cataforico del trapassato italiano: un espediente testuale per la messa in rilievo.» Linguistica 28/2, 183-195.

MikLIČ, Tjaša (2003a) «Interpretazione della funzione testuale dei paradigmi verbali italiani. Tentativo di un modello di analisi integrata.» In: M. G. Marcellesi/A. Rocchetti (a cura di), Il verbo italiano. Roma: Bulzoni, 553-570.

MikLIČ, Tjaša (2003b) «Il discorso indiretto libero nel romanzo di Giorgio Bassani Il giardino dei Finzi-Contini: funzioni testuali e caratteristiche linguistiche.» Linguistica 43, 93-108.

MıkLIČ, Tjaša (2004) «Testi narrativi, azioni centrali e paradigmi verbali.» In: P. D'Achille (a cura di), Generi, architetture e forme testuali. Firenze: F. Cesati, 145-160.

MikLIČ, Tjaša (2007) «Metafore o načinih gledanja na zunajjezikovna dejanja v obravnavanju glagolskega vida.» Slavistična revija 55/1-2, 85-103.

MikLIČ, Tjaša/Martina OŽBOT (2001) «L'insegnamento dell'italiano in Slovenia.» Bulletin VALS-ASLA 73, 113-121.

MikLIČ, Tjaša/Martina OŽBOT (2007) «Teaching the uses of Italian verb forms to Slovene speakers.» Linguistica 47, 65-76. 
Оžвот, Martina (2009) «Nekaj kontrastivnih beležk o italiajanščini in slovenščini in nekaj opažanj o jezikovni produkciji pri govorcih slovenščine v Italiji.» Jezik in slovstvo 54/1, 25-47.

SICHERL, Eva (1998) «Interferenca pri rabi angleških predlogov na osnovi testiranj med študenti anglistike.» In: I. Štrukelj (a cura di), Jezik za danes in jutri. Ljubljana: Društvo za uporabno jezikoslovje, 356-368.

SKela, Janez (a cura di) (2008) Učenje in poučevanje tujih jezikov na Slovenskem. Ljubljana: Tangram. ŠIFRAR KALAN, Marjana (2008) «Raziskovanje učnih strategij za usvajanje besedišča.» In: J. Skela (a cura di), 52-73.

Študijski program Prevajalski študij angleščina-nemščina/francoščina/italijanščina, nemščinaangleščina/ francoščina/italijanščina. Ljubljana (2004): Univerza v Ljubljani Filozofska fakulteta Oddelek za prevajalstvo.

TOPORIŠIČ, Jože $\left({ }^{4} 2000\right)$ Slovenska slovnica. Maribor: Obzorja.

WANDRUSZKA, Ulrich (2001) «Frasi subordinate al congiuntivo.» In: L. Renzi/G. Salvi/A. Cardinaletti (a cura di), Grande grammatica italiana di consultazione. Vol. III. Bologna: il Mulino, 415- 481.

\section{Siti internet}

http://dev.sslmit.unibo.it/corpora/query.php? $\operatorname{mode}=$ simple\&path=\&name=Repubblica. Data di consultazione: 13. 5. 2009.

http://www.prevajalstvo.net/. Data di consultazione: 7. 4. 2009.

http://www.prevajalstvo.net/files/Medjezikovno\%20posredovanje_predstavitveni.pdf. Data di consultazione: 7. 4. 2009.

http://www.ff.uni-lj.si/oddelki/romanistika/IT_star_pravi.htm. Data di consultazione: 7. 4. 2009.

\section{APPENDICE}

La Tabella 5 riassume i risultati principali delle analisi. È stata elaborata in modo tale da disporre le soluzioni degli studenti in un ordine che tiene conto del grado di criticità di ciascun fenomeno: si va, cioè, da quelli con il maggior numero di risposte errate a quelli con il maggior numero di soluzioni corrette (colonna 1). Nella colonna 2 è indicato il fenomeno con la soluzione corretta e, se necessario, anche con una soluzione parzialmente corretta; 80 nella colonna 3 , invece, è evidenziata la posizione del fenomeno nel rispettivo cloze (ad es. A.1, A.2, B.5 ecc.). Nella colonna 4 è indicata la percentuale complessiva delle soluzioni errate per ciascuno dei fenomeni analizzati, a cui fanno seguito i dati parziali relativi alle soluzioni inappropriate degli studenti rispettivamente di Italianistica (IT) e di Traduzione (TRA). I segni $\uparrow, \downarrow, \sim,=$ accanto alle percentuali evidenziano il rapporto interno tra le percentuali di soluzioni inappropriate di ogni singolo sottogruppo. Quando all'interno di uno dei due gruppi la prestazione migliora a seconda dell'anno di corso frequentato ${ }^{81}$ il rapporto interno viene segnalato con il simbolo $\uparrow$. La

80 Con i simboli $\uparrow$ e $\downarrow$ nella colonna 2 si desidera mostrare la correlazione tra la prestazione negli esercizi 2D, 2E e 2GFC (il secondo tentativo) con la prestazione nei corrispettivi esercizi D, E e GFC.

81 Con «miglioramento della prestazione in dipendenza dagli anni di studio» si intendono situazioni in cui il sottogruppo IT2/TRA2 raggiunge la percentuale più alta di soluzioni innapropriate, il sottogruppo IT3/TRA3 una percentuale più bassa e il sottogruppo IT4/TRA4 la percentuale più bassa di tali soluzioni. 
situazione opposta è evidenziata con il simbolo $\downarrow$. Se le percentuali delle soluzioni inappropriate con il progredire degli anni di studio rimangono uguali il rapporto è evidenziato con il simbolo =. Quando invece non c'è una correlazione né positiva né negativa tra il miglioramento della prestazione e gli anni di studio il rapporto è evidenziato con il simbolo .82 Tra le soluzioni inappropriate una o due compaiono con maggiore frequenza. La prima percentuale della colonna 5 indica appunto la media delle percentuali dei gruppi IT e TRA relative alla soluzione inappropriata più frequente. Sotto la media sono indicati i dati parziali dei gruppi IT e TRA. Tra parentesi, accanto all'indicazione della forma verbale usata con più frequenza, viene evidenziata la maggiore o minore presenza di interferenze. Con il simbolo + è indicata l'interferenza totale, con il simbolo / l'interferenza parziale. Le sigle $s$ (sloveno), sn (sloveno in combinazione con l'italiano del Nord d'Italia), st (strutture di superficie del testo sloveno) e snt (strutture di superficie del testo sloveno in combinazione con l'influsso dell'italiano del Nord d'Italia) si riferiscono all'origine dell'interferenza. Nella colonna 6 sono indicate le soluzioni che nei cloze degli studenti compaiono con minore frequenza, con i rispettivi tipi di interferenza. Con ogni percentuale è indicata la quantità di un determinato tipo di soluzioni rispetto all'insieme di tutte le soluzioni (appropriate, inappropriate e parzialmente corrette). ${ }^{83}$

\begin{tabular}{|c|c|c|c|c|c|}
\hline $\begin{array}{l}1 \\
\%\end{array}$ & 2 & 3 & $\begin{array}{l}4 \\
\%\end{array}$ & $\begin{array}{l}5 \\
\%\end{array}$ & 6 \\
\hline \multirow{4}{*}{$50-100$} & $\begin{array}{l}\text { FB (anteriorità nell'ambito della } \\
\text { frase indipendente) } \\
\text { era stata (TP) }\end{array}$ & GFC. 8 & $\begin{array}{l}97,14 \\
=100 \text { IT } \\
\uparrow 94,29 \text { TRA }\end{array}$ & $\begin{array}{l}\text { IM (s+): } 71,43 \\
82,86 \text { IT; } 60 \text { TRA }\end{array}$ & $\mathrm{Pr}^{\prime}, \mathrm{IM}$ ', PR (s/) \\
\hline & $\begin{array}{l}\text { FB (anteriorità nell'ambito della } \\
\text { frase indipendente) } \\
\text { era stata (TP) } \\
\uparrow\end{array}$ & 2GFC. 8 & $\begin{array}{l}94,29 \\
\sim 94,29 \text { IT } \\
\uparrow 94,29 \text { TRA }\end{array}$ & $\begin{array}{l}\text { IM (s+): } 61,43 \\
62,86 \text { IT; } 60 \text { TRA }\end{array}$ & $\begin{array}{l}\text { IM', TP', CC, } \\
\text { PP (s/), PR (s/) }\end{array}$ \\
\hline & $\begin{array}{l}\text { FB (anteriorità nell'ambito della } \\
\text { frase indipendente) } \\
\text { ero entrato (TP) }\end{array}$ & GFC. 5 & $\begin{array}{l}94,29 \\
\uparrow 91,43 \text { IT } \\
\sim 97,14 \text { TRA }\end{array}$ & $\begin{array}{l}\text { PR (s+): 66,2 } \\
60 \text { IT; } 74,29 \text { TRA }\end{array}$ & PP $\left(\mathrm{s}^{+}\right), \mathrm{IM}, \mathrm{TR}$ \\
\hline & $\begin{array}{l}\text { FB (anteriorità nell'ambito della } \\
\text { frase indipendente) } \\
\text { ero entrato (TP) } \\
=\end{array}$ & 2GFC. 5 & $\begin{array}{l}94,29 \\
\uparrow 91,43 \text { IT } \\
\sim 97,14 \text { TRA }\end{array}$ & $\begin{array}{l}\text { PR (s+): } 65,71 \\
62,86 \text { IT; } 68,57 \\
\text { TRA }\end{array}$ & PP (s+), IM \\
\hline
\end{tabular}

${ }^{82} \mathrm{Si}$ tratta di situazioni in cui i sottogruppi sono disposti in base alla quantità di soluzioni inappropriate - dal grado massimo a quello minimo - in uno dei seguenti modi: IT2/TRA2 > IT4/TRA4 > IT3/TRA3, IT3/TRA3 > IT4/TRA4 > IT2/TRA2, IT4/TRA4 > IT2/TRA2 > IT3/TRA3, IT3/TRA3 > IT2/TRA2 > IT4/TRA4.

${ }^{83}$ La Tabella 5 contiene anche alcuni altri simboli. Ø: assenza della soluzione; $1 \frac{1}{2}$ Ø: assenza parziale della soluzione; (e): errore (il simbolo (e) accompagna le forme verbali che potrebbero essere considerate corrette o parzialmente corrette se non presentassero al contempo un altro tipo di errore: ad. es. al posto di non si svolsero in E.1 è usata la forma non si svolse, non furono svolte); (a) e (p): diatesi attiva e passiva (i due simboli sono usati soltanto nei casi in cui una tale distinzione è necessaria: ad. es. in D.6, che prevede l'impiego della forma passiva dell'IM' fosse chiamato, è importante distinguere tale uso dall'uso errato della forma attiva dello stesso paradigma come chiamassero o chiamasse). 


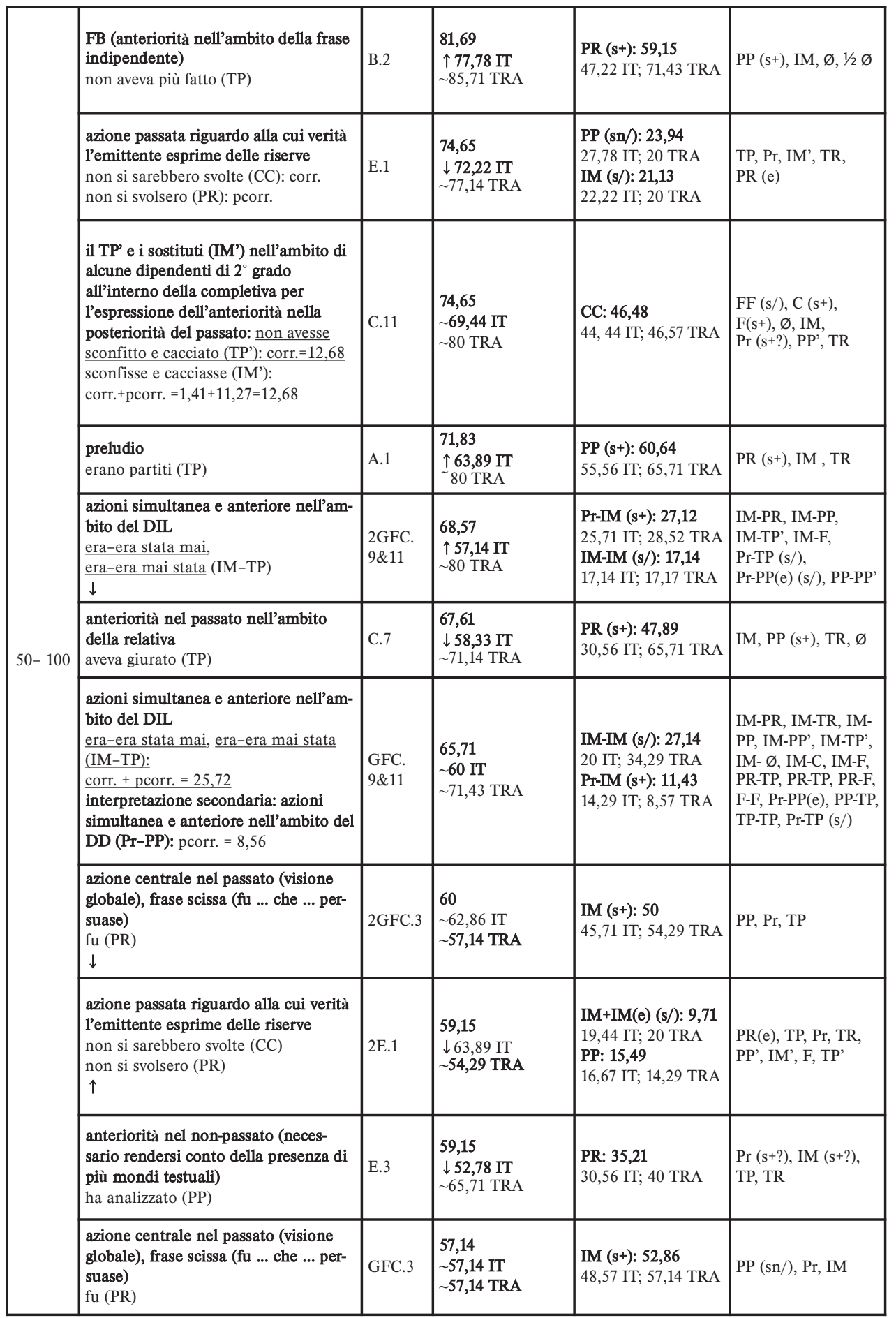




\begin{tabular}{|c|c|c|c|c|c|}
\hline \multirow{9}{*}{$50-100$} & $\begin{array}{l}\text { anteriorità nel passato nell'ambito } \\
\text { della relativa } \\
\text { aveva chiesto (TP) }\end{array}$ & D.5 & $\begin{array}{l}54,93 \\
\sim 44,44 \text { IT } \\
\sim 65,71 \text { TRA }\end{array}$ & $\begin{array}{l}\text { PR (s/): } 42,25 \\
27,78 \text { IT; } 57,14 \text { TRA }\end{array}$ & $\begin{array}{l}\text { IM (s+), PP (s/), } \\
\text { TR, TP', PP', G }\end{array}$ \\
\hline & $\begin{array}{l}\text { azione centrale nel passato (necessario } \\
\text { rendersi conto della presenza di più } \\
\text { mondi testuali) } \\
\text { attribuirono (PR) } \\
\downarrow\end{array}$ & $2 \mathrm{E} .7$ & $\begin{array}{l}\mathbf{5 4 , 9 3} \\
\sim 58,33 \text { IT } \\
\downarrow 51,43 \text { TRA }\end{array}$ & $\begin{array}{l}\text { PP(sn+): } \mathbf{2 8 , 1 7} \\
\text { 22,22 IT; } 34,29 \text { TRA }\end{array}$ & $\begin{array}{l}\text { TP, TR, Pr, F, IM, } \\
\text { IM(p), IM' }\end{array}$ \\
\hline & $\begin{array}{l}\text { il TP' e i sostituti (IM') nell'ambito di } \\
\text { alcune dipendenti di } 2^{\circ} \text { grado all'interno } \\
\text { della completiva per l'espressione dell'an- } \\
\text { teriorità nella posteriorità del passato } \\
\text { avesse vendicato }\left(\mathrm{TP}^{\prime} \text { ') + vendicasse }\right. \\
\text { (IM'): corr. }\end{array}$ & C. 8 & $\begin{array}{l}54,93 \\
\downarrow 50 \text { IT } \\
\sim 60 \text { TRA }\end{array}$ & $\begin{array}{l}\text { CC: } 36,62 \\
36,11 \text { IT; } 34,17 \text { TRA }\end{array}$ & $\begin{array}{l}\mathrm{FF}(\mathrm{s} /), \mathrm{F}\left(\mathrm{s}^{+}\right), \varnothing \\
\mathrm{C}\left(\mathrm{s}^{+}\right), \mathrm{PP}^{\prime}\end{array}$ \\
\hline & $\begin{array}{l}\text { anteriorità nel passato, nell'ambito } \\
\text { della relativa } \\
\text { aveva chiesto (TP) } \\
\uparrow\end{array}$ & 2D.5 & $\begin{array}{l}53,52 \\
\sim 44,44 \text { IT } \\
\downarrow 62,86 \text { TRA }\end{array}$ & $\begin{array}{l}\text { PR (s/): 40,84 } \\
\text { 27,78 IT; 54,29 TRA }\end{array}$ & $\begin{array}{l}\text { IM (s+), Pr, PP (s/), } \\
\text { TR, PP', TP' }\end{array}$ \\
\hline & $\begin{array}{l}\text { simultaneità nel passato (visione } \\
\text { corsiva) } \\
\text { era determinato (IM): corr. } \\
\text { sarebbe stato determinato (CC): pcorr. }\end{array}$ & C.10 & $\begin{array}{l}53,52 \\
\sim 50 \text { IT } \\
\downarrow 57,14 \text { TRA }\end{array}$ & $\begin{array}{l}\text { PR (s+?): 40,85 } \\
\text { 33,33 IT; 48,57 TRA }\end{array}$ & $\begin{array}{l}\mathrm{PP}(\mathrm{sn} / ?), \mathrm{PP} \text { ', TP, } \\
\mathrm{IM} \text { ' }\end{array}$ \\
\hline & $\begin{array}{l}\text { anteriorità nel passato, dipendente } \\
\text { implicita } \\
\text { avermi introdotto (INFF) }\end{array}$ & GFC.1 & $\begin{array}{l}52,86 \\
\sim 48,57 \text { IT } \\
\downarrow 57,14 \text { TRA }\end{array}$ & $\begin{array}{l}\text { INFF(e): } 22,86 \\
\text { 17,14 IT; } 28,57 \text { TRA }\end{array}$ & $\begin{array}{l}\text { INF, G, GG, } \\
\text { INFF(e), TP, TP', }, \\
\text { PP (st+), PR (st+) }\end{array}$ \\
\hline & $\begin{array}{l}\text { anteriorità nel non-passato, } \\
\text { (necessario rendersi conto della } \\
\text { presenza di più mondi testuali) } \\
\text { ha analizzato (PP) } \\
\uparrow\end{array}$ & $2 \mathrm{E} .3$ & $\begin{array}{l}52,11 \\
\sim 47,22 \text { IT } \\
\sim 57,14 \text { TRA }\end{array}$ & $\begin{array}{l}\text { PR: } 38,03 \\
\text { 38,89 IT; } 37,14 \text { TRA }\end{array}$ & $\mathrm{IM}(\mathrm{s}+?), \operatorname{Pr}, \mathrm{TP}$ \\
\hline & $\begin{array}{l}\text { simultaneità nel passato (visione } \\
\text { corsiva) } \\
\text { veniva (IM) } \\
\downarrow\end{array}$ & $2 \mathrm{D} .3$ & $\begin{array}{l}50,70 \\
\uparrow 52,78 \mathrm{IT} \\
\sim 48,57 \mathrm{TRA}\end{array}$ & $\begin{array}{l}\operatorname{Pr}(\mathrm{s}+): 18,31 \\
\text { 19,44 IT; } 7,14 \text { TRA }\end{array}$ & $\mathrm{IM}(\mathrm{e}), \mathrm{IM}$ ', PR \\
\hline & $\begin{array}{l}\text { azione centrale nel passato (necessario } \\
\text { rendersi conto della presenza di più } \\
\text { mondi testuali) } \\
\text { attribuirono }(\mathrm{PR})\end{array}$ & E.7 & $\begin{array}{l}50,70 \\
\uparrow 50 \text { IT } \\
\uparrow 51,43 \mathrm{TRA}\end{array}$ & $\begin{array}{l}\text { PP (sn+): } 19,71 \\
\text { 13,89 IT; } 25,71 \text { TRA }\end{array}$ & $\begin{array}{l}\text { TP, TR, IM, F, Pr, } \\
\text { IM' }\end{array}$ \\
\hline \multirow{5}{*}{$40-50$} & $\begin{array}{l}\text { simultaneità nel passato (visione } \\
\text { corsiva) } \\
\text { veniva (IM) }\end{array}$ & D. 3 & $\begin{array}{l}49,3 \\
\uparrow 50 \text { IT } \\
\sim 48,57 \text { TRA }\end{array}$ & $\begin{array}{l}\operatorname{Pr}\left(\mathbf{s}^{+}+\right): 16,90 \\
\text { 19,44 IT; } 14,29 \text { TRA }\end{array}$ & $\mathrm{IM}(\mathrm{e}), \mathrm{IM}^{\prime}, \mathrm{PR}$ \\
\hline & $\begin{array}{l}\text { azione extratemporale, esclusione di } \\
\text { altre interpretazioni con l'osservazione } \\
\text { attenta del cotesto } \\
\text { sostiene (Pr) }\end{array}$ & E.2 & $\begin{array}{l}49,3 \\
\uparrow 50 \text { IT } \\
\sim 48,57 \text { TRA }\end{array}$ & $\begin{array}{l}\text { IM (s+): } 35,21 \\
\text { 27,78 IT; 42,86 TRA }\end{array}$ & $\begin{array}{l}\mathrm{PR}, \mathrm{PP}\left(\mathrm{s}^{+}\right), \\
\mathrm{PP}(\mathrm{p})(\mathrm{s} /), \mathrm{TR}, \mathrm{TP}\end{array}$ \\
\hline & $\begin{array}{l}\text { FB (anteriorità nell'ambito della frase } \\
\text { indipendente) } \\
\text { si era allontanata (TP) }\end{array}$ & B.1 & $\begin{array}{l}47,89 \\
\uparrow 36,11 \text { IT } \\
\sim 60 \text { TRA }\end{array}$ & $\begin{array}{l}\text { PR (s+): } 33,80 \\
\text { 19,44 IT; } 48,57 \text { TRA }\end{array}$ & $\mathrm{PP}\left(\mathrm{s}^{+}\right), \mathrm{TR}, \mathrm{PP}$ \\
\hline & $\begin{array}{l}\text { azione extratemporale } \\
\text { ricordano }(\mathrm{Pr})\end{array}$ & E.4 & $\begin{array}{l}45,07 \\
\uparrow 52,78 \text { IT } \\
\uparrow 37,14 \text { TRA }\end{array}$ & $\begin{array}{l}\text { IM (s+?): } 33,80 \\
\text { 36,11 IT; } 11,43 \text { TRA }\end{array}$ & $\operatorname{Pr}(\mathrm{e}), \mathrm{PP}, \mathrm{IM}, \mathrm{PR}, \varnothing$ \\
\hline & $\begin{array}{l}\text { simultaneità nel passato (visione } \\
\text { corsiva) } \\
\text { intendeva (IM) }\end{array}$ & C. 6 & $\begin{array}{l}43,66 \\
\sim 41,67 \text { IT } \\
\sim 45,71 \text { TRA }\end{array}$ & $\begin{array}{l}\text { PR: } 40,85 \\
\text { 38,89 IT; } 42,86 \text { TRA }\end{array}$ & $\varnothing, \mathrm{TR}, \mathrm{TP}$ \\
\hline
\end{tabular}




\begin{tabular}{|c|c|c|c|c|c|}
\hline & $\begin{array}{l}\text { posteriorità nel passato nell'ambito } \\
\text { della completiva in seguito al verbo } \\
\text { volitivo decidere nella principale } \\
\text { avrebbe dato (CC)/fosse dato (IM') } \\
\downarrow\end{array}$ & 2D.1 & $\begin{array}{l}42,26 \\
\sim 44,44 \text { IT } \\
\uparrow 40 \text { TRA }\end{array}$ & $\begin{array}{l}\mathbf{F}\left(\mathbf{s}^{+}\right): \mathbf{1 5}, 49 \\
13,89 \text { IT; } 17,41 \text { TRA }\end{array}$ & $\begin{array}{l}\mathrm{PR}, \mathrm{IM}^{\prime}, \mathrm{C}, \mathrm{Pr}\left(\mathrm{s}^{+}\right), \\
\mathrm{FF}\end{array}$ \\
\hline \multirow{9}{*}{$30-40$} & $\begin{array}{l}\text { posteriorità nel passato nell'ambito } \\
\text { della completiva in seguito al verbo } \\
\text { volitivo decidere nella principale } \\
\text { avrebbe dato }(\mathrm{CC}) / \text { fosse dato (IM') }\end{array}$ & D.1 & $\begin{array}{l}39,44 \\
\uparrow 44,44 \text { IT } \\
\sim 34,29 \text { TRA }\end{array}$ & $\begin{array}{l}\mathbf{F}(\mathbf{s}+): \mathbf{1 4 , 0 8} \\
13,89 \text { IT; } 14,29 \text { TRA }\end{array}$ & $\begin{array}{l}\operatorname{Pr}\left(\mathrm{s}^{+}\right), \mathrm{PR}, \mathrm{IM}^{\prime}, \mathrm{C}, \\
\mathrm{FF}, \mathrm{TP}\end{array}$ \\
\hline & $\begin{array}{l}\text { posteriorità nel passato in } \\
\text { combinazione con l'espressione del } \\
\text { desiderio nell'ambito della } \\
\text { completiva in seguito al verbo volitivo } \\
\text { chiedere nella principale } \\
\text { fosse chiamato/venisse chiamato } \\
\text { (IM') }\end{array}$ & D.6 & $\begin{array}{l}38,03 \\
\uparrow 41,67 \text { IT } \\
\sim 34,29 \text { TRA }\end{array}$ & $\begin{array}{l}\text { IM'(e, a): } 9,86 \\
\text { 13,89 IT; } 5,71 \text { TRA }\end{array}$ & $\begin{array}{l}\text { C(a), } \operatorname{Pr}^{\prime}(p)\left(s^{+}\right) \\
\operatorname{Pr}^{\prime}(a)(s t+) \\
\operatorname{PR}(a)(s t /) \\
I^{\prime}(a)(s t+), \\
\text { IM (p), C(p), CC(p), } \\
\operatorname{Pr}^{\prime}(p), \operatorname{PR}(p), \\
\text { TP'(p), } 1 / 2 \varnothing, F F \text { (a) }\end{array}$ \\
\hline & $\begin{array}{l}\text { posteriorità nel passato in } \\
\text { combinazione con l'espressione del } \\
\text { desiderio nell'ambito della completiva } \\
\text { in seguito al verbo volitivo chiedere } \\
\text { nella principale } \\
\text { fosse chiamato/venisse chiamato (IM') } \\
=\end{array}$ & 2D.6 & $\begin{array}{l}36,62 \\
\uparrow 41,67 \text { IT } \\
\sim 31,43 \text { TRA }\end{array}$ & $\begin{array}{l}\text { IM' (e, a): } 9,86 \\
\text { 13,89 IT; 5,71 TRA }\end{array}$ & $\begin{array}{l}\text { C(a), } \operatorname{Pr}^{\prime}(a)\left(s^{+}\right), \\
\text {PART, PR(a), } \\
\text { IM'(a) (st+), IM, C(p), } \\
\text { CC(p), Pr'(p), PR(p), } \\
\text { TP'(p), 1/2 Ø, FF(a) }\end{array}$ \\
\hline & $\begin{array}{l}\text { tendenza a mantenere l'ottica } \\
\text { costante nell'ambito della } \\
\text { coordinazione copulativa } \\
\text { scrissero/paragonarono (PR): corr.+ } \\
\text { pcorr. }=14,08+4,23=18,31 \\
\text { scrivevano/paragonavano (IM): corr.+ } \\
\text { pcorr. }=45,07\end{array}$ & C. $3 / 5$ & $\begin{array}{l}36,62 \\
\uparrow 33,33 \text { IT } \\
\sim 40 \text { TRA }\end{array}$ & $\begin{array}{l}\text { PR-IM (s+): } \mathbf{2 6 , 7 6} \\
\text { 22,22 IT; 31,43 TRA }\end{array}$ & $\begin{array}{l}\text { PP-IM (s+), TP-PR, } \\
\text { PR-IM' }\end{array}$ \\
\hline & $\begin{array}{l}\text { anteriorità nel passato nell'ambito } \\
\text { dell'interrogativa indiretta introdotta } \\
\text { da chiedere nella principale } \\
\text { avesse aspettato (TP') (corr.+pcorr. = } \\
\begin{array}{l}18,18) \\
\text { aveva aspettato (TP) (corr.+pcorr. }= \\
46,59)\end{array}\end{array}$ & B. 4 & $\begin{array}{l}35,21 \\
\uparrow 33,33 \text { IT } \\
\sim 37,14 \text { TRA }\end{array}$ & $\begin{array}{l}\text { IM (s+): } 12,86 \\
8,33 \text { IT; } 17,14 \text { TRA } \\
\text { PR (s/): } 8,45 \\
\text { 5,56 IT; } 11,43 \text { TRA }\end{array}$ & $\begin{array}{l}\mathrm{IM}^{\prime}(\mathrm{s}+), \mathrm{TR}, \mathrm{PP}(\mathrm{s} /) \\
\mathrm{PP}^{\prime}(\mathrm{s} /), \mathrm{TP}(\mathrm{e}), 1 / 2 \varnothing\end{array}$ \\
\hline & $\begin{array}{l}\text { azione extratemporale, esclusione di } \\
\text { altre interpretazioni con } \\
\text { l'osservazione attenta del cotesto } \\
\text { sostiene (Pr) } \\
\uparrow\end{array}$ & $2 \mathrm{E} .2$ & $\begin{array}{l}35,21 \\
\uparrow 38,39 \text { IT } \\
\sim 31,43 \text { TRA }\end{array}$ & $\begin{array}{l}\text { IM: } 26,76 \\
25 \text { IT; } 28,57 \text { TRA }\end{array}$ & PR, TP, PP, PP (p) \\
\hline & $\begin{array}{l}\text { parte del FB: } \\
\text { avevo notato (TP) } \\
\text { commento: } \\
\text { notai (PR) }\end{array}$ & 2GFC.7 & $\begin{array}{l}34,29 \\
\uparrow 34,29 \text { IT } \\
\sim 34,29 \text { TRA }\end{array}$ & $\begin{array}{l}\text { PP (s+): } 17,14 \\
20 \text { IT; } 14,29 \text { TRA } \\
\text { TP (e): } 15,71 \\
11,43 \text { IT; } 20 \text { TRA }\end{array}$ & PR (e) \\
\hline & 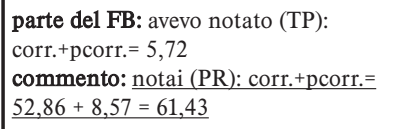 & GFC.7 & $\begin{array}{l}32,86 \\
\uparrow 37,14 \text { IT } \\
\uparrow 28,57 \text { TRA }\end{array}$ & $\begin{array}{l}\text { PP (s+): } 17,14 \\
22,86 \text { IT; } 11,43 \text { TRA }\end{array}$ & $\mathrm{PR}(\mathrm{e}), \mathrm{TP}(\mathrm{e})$ \\
\hline & $\begin{array}{l}\text { azione presentata sia come un'azione } \\
\text { di secondo piano nel passato (visione } \\
\text { corsiva), oppure come extratemporale } \\
\text { indicava (IM); indica (Pr) }\end{array}$ & E. 5 & $\begin{array}{l}30,99 \\
\uparrow 36,11 \text { IT } \\
\sim 25,71 \text { TRA }\end{array}$ & $\begin{array}{l}\text { PR (s+?): } 19,71 \\
\text { 19,44 IT; } 20 \text { TRA }\end{array}$ & $\mathrm{IM}(\mathrm{e}), \mathrm{TP}, \mathrm{PP}(\mathrm{sn}+?)$ \\
\hline
\end{tabular}




\begin{tabular}{|c|c|c|c|c|c|}
\hline $30-40$ & $\begin{array}{l}\text { azione presentata sia come un'azione } \\
\text { di secondo piano nel passato (visione } \\
\text { corsiva), oppure come extratemporale } \\
\text { indicava (IM); indica (Pr) } \\
=\end{array}$ & 2E. 5 & $\begin{array}{l}30,99 \\
\uparrow 36,11 \text { IT } \\
\sim 25,71 \text { TRA }\end{array}$ & $\begin{array}{l}\text { PR: } 19,72 \\
\text { 16,67 IT; } 22,86 \text { TRA }\end{array}$ & $\mathrm{IM}(\mathrm{e}), \mathrm{TP}, \mathrm{PP}$ \\
\hline \multirow{12}{*}{$20-30$} & $\begin{array}{l}\text { azione centrale nel passato (visione } \\
\text { globale) } \\
\text { trasgredì (PR) }\end{array}$ & D. 4 & $\begin{array}{l}29,58 \\
\uparrow 38,89 \text { IT } \\
\sim 20 \text { TRA }\end{array}$ & $\begin{array}{l}\text { PR(e): } 11,27 \\
\text { 16,67 IT; 5,71 TRA }\end{array}$ & $\mathrm{PP}\left(\mathrm{sn}^{+}\right), \mathrm{Pr}, \mathrm{TP}, \varnothing, \mathrm{CC}$ \\
\hline & $\begin{array}{l}\text { azione centrale nel passato (visione } \\
\text { globale) } \\
\text { trasgredì (PR) }\end{array}$ & 2D.4 & $\begin{array}{l}29,58 \\
\uparrow 36,11 \text { IT } \\
\sim 22,86 \text { TRA }\end{array}$ & $\begin{array}{l}\text { PR(e): } 11,27 \\
\text { 16,67 IT; 5,71 TRA }\end{array}$ & $\mathrm{PP}(\mathrm{sn}+), \mathrm{Pr}, \mathrm{TP}, \mathrm{CC}$ \\
\hline & $\begin{array}{l}\text { azione centrale contigua nel passato } \\
\text { (PR)/azione anteriore nel passato (TP) } \\
\text { chiese (PR): } 63,38 \\
\text { aveva chiesto (TP): } 7,04\end{array}$ & B. 3 & $\begin{array}{l}29,58 \\
\uparrow 44,44 \text { IT } \\
\uparrow 14,29 \text { TRA }\end{array}$ & $\begin{array}{l}\text { PP (sn+): } 21,13 \\
\text { 30,56 IT; } 11,43 \text { TRA }\end{array}$ & IM', IM (s+?), PP' \\
\hline & $\begin{array}{l}\text { anteriorità nel passato, dipendente } \\
\text { implicita } \\
\text { avermi introdotto (INFF) } \\
\uparrow\end{array}$ & 2GFC.1 & $\begin{array}{l}28,57 \\
\uparrow 31,43 \text { IT } \\
\downarrow 25,71 \text { TRA }\end{array}$ & soluzioni disperse & $\begin{array}{l}\text { INF, GG, INFF, } \\
\text { PP (st+), TP, TP', } \varnothing, \\
\text { PR (st+) }\end{array}$ \\
\hline & $\begin{array}{l}\text { FB (anteriorità nell'ambito della frase } \\
\text { indipendente) } \\
\text { (li) aveva estratti (TP) }\end{array}$ & A. 4 & $\begin{array}{l}28,18 \\
\uparrow 27,78 \mathrm{IT} \\
\uparrow 28,57 \mathrm{TRA}\end{array}$ & $\begin{array}{l}\text { PP (s+): } 28,18 \\
27,78 \text { IT; } 28,57 \text { TRA }\end{array}$ & $\begin{array}{l}\text { assenza di soluzioni } \\
\text { inappropriate meno } \\
\text { frequenti }\end{array}$ \\
\hline & $\begin{array}{l}\text { azione extratemporale } \\
\text { ricordano }(\mathrm{Pr})\end{array}$ & 2E. 4 & $\begin{array}{l}28,17 \\
\uparrow 38,89 \text { IT } \\
\uparrow 17,14 \text { TRA }\end{array}$ & $\begin{array}{l}\text { IM: } 16,90 \\
\text { 22,22 IT; } 11,43 \text { TRA }\end{array}$ & $\operatorname{Pr}(\mathrm{e}), \mathrm{IM}(\mathrm{e}), \mathrm{IM}^{\prime}, \mathrm{PP}, \varnothing$ \\
\hline & $\begin{array}{l}\text { simultaneità nel passato, azione } \\
\text { verbalizzata nell'ambito del discorso } \\
\text { indiretto, visione corsiva } \\
\text { somigliava (IM) }\end{array}$ & C. 4 & $\begin{array}{l}26,76 \\
\sim 30,56 \text { IT } \\
\sim 22,86 \text { TRA }\end{array}$ & $\begin{array}{l}\text { IM': } 19,72 \\
27,78 \text { IT; } 11,43 \text { TRA }\end{array}$ & $\operatorname{Pr}\left(\mathrm{s}^{+}\right)$ \\
\hline & $\begin{array}{l}\text { costrutto implicito } \\
\text { migrati (PART) }\end{array}$ & E.6 & $\begin{array}{l}23,94 \\
\sim 11,11 \text { IT } \\
\sim 37,14 \text { TRA }\end{array}$ & $\begin{array}{l}\text { PR (st+): 5,63 } \\
\text { 5,56 IT; 5,71 TRA }\end{array}$ & $\begin{array}{l}\text { PARTPr, G, GG, IM, } \\
\text { PP(snt }+ \text { ), TP, Ø }\end{array}$ \\
\hline & $\begin{array}{l}\text { visione corsiva dell'azione passata } \\
\text { verbalizzata nell'ambito della frase } \\
\text { citante intercalata al DIL: } \\
\text { dicevo (IM) } \\
\text { visone globale dell'azione passata } \\
\text { verbalizzata nell'ambito della frase } \\
\text { citante intercalata al DIL } \\
\text { dissi (PR) } \\
\downarrow\end{array}$ & 2GFC.10 & $\begin{array}{l}22,86 \\
\uparrow 22,86 \text { IT } \\
\sim 22,86 \text { TRA }\end{array}$ & $\begin{array}{l}\text { PP (sn+): } 17,14 \\
17,14 \text { IT; } 17,14 \text { TRA }\end{array}$ & $\begin{array}{l}\text { F, Pr, PP/PR, IM', } \\
\text { PR(e) }\end{array}$ \\
\hline & $\begin{array}{l}\text { visione corsiva dell'azione passata } \\
\text { verbalizzata nell'ambito della frase } \\
\text { citante intercalata al DIL: } \\
\text { dicevo (IM): corr. }=25,71 \\
\text { visone globale dell'azione passata } \\
\text { verbalizzata nell'ambito della frase } \\
\text { citante intercalata al DIL } \\
\text { dissi (PR): corr. }{ }^{+} \text {pcorr. }=52,86\end{array}$ & $\begin{array}{l}\text { GFC. } \\
10\end{array}$ & $\begin{array}{l}21,43 \\
\uparrow 31,43 \text { IT } \\
\downarrow 11,43 \text { TRA }\end{array}$ & $\begin{array}{l}\text { PP (sn+): } 8,57 \\
11,43 \text { IT; } 5,71 \text { TRA }\end{array}$ & $\mathrm{PR}(\mathrm{e}), \operatorname{IM}(\mathrm{e}), \mathrm{C}, \operatorname{Pr}, \mathrm{F}, \varnothing$ \\
\hline & $\begin{array}{l}\text { simultaneità nella posteriorità nel } \\
\text { passato } \\
\text { sorrideva (IM) }\end{array}$ & GFC.2 & $\begin{array}{l}21,43 \\
\sim 20 \text { IT } \\
\sim 22,86 \text { TRA }\end{array}$ & $\begin{array}{l}\text { PR (s+): } 12,86 \\
\text { 11,43 IT; } 14,29 \text { TRA }\end{array}$ & $\operatorname{Pr}\left(\mathrm{s}^{+}\right), \mathrm{CC}, \mathrm{TP}, \varnothing, \mathrm{IM}^{\prime}$ \\
\hline & $\begin{array}{l}\text { costrutto implicito } \\
\text { migrati (PART) } \\
\uparrow\end{array}$ & 2E.6 & $\begin{array}{l}21,13 \\
\sim 8,33 \text { IT } \\
\sim 34,29 \text { TRA }\end{array}$ & $\begin{array}{l}\text { PR (st+): 7,04 } \\
\text { 5,56 IT; 8,57 TRA }\end{array}$ & $\begin{array}{l}\text { PARTPr, G, GG, IM, } \\
\text { PP (snt }+ \text { ), TP }\end{array}$ \\
\hline
\end{tabular}




\begin{tabular}{|c|c|c|c|c|c|}
\hline \multirow{7}{*}{$10-20$} & $\begin{array}{l}\text { simultaneità nel passato, visione } \\
\text { corsiva } \\
\text { erano (IM) }\end{array}$ & A. 3 & $\begin{array}{l}\mathbf{1 9 , 7 2} \\
\uparrow 22,22 \text { IT } \\
\sim 17,14 \text { TRA }\end{array}$ & $\begin{array}{l}\text { PP: } 12,58 \\
\text { 19,44 IT; } 5,71 \text { TRA }\end{array}$ & TP, PR \\
\hline & $\begin{array}{l}\text { simultaneità nel passato, visione } \\
\text { corsiva scandalizzava (IM) }(38,03) \\
\text { contiguità, azione centrale nel } \\
\text { passato: scandalizzò }(\mathrm{PR})(43,66)\end{array}$ & C. 2 & $\begin{array}{l}18,31 \\
\uparrow 22,22 \text { IT } \\
\sim 14,29 \text { TRA }\end{array}$ & $\begin{array}{l}\text { PR(e) (st+) + } \\
\text { IM(e) (st+) + } \\
\text { PP(p) (snt }+): 8,45 \\
\text { 16,67 IT; 0 TRA }\end{array}$ & TP, PP (sn+) \\
\hline & $\begin{array}{l}\text { posteriorità nel passato } \\
\text { nell'ambito delle completive } \\
\text { tornasse (IM'): } 38,03 \\
\text { sarebbe tornata (CC): } 43,67\end{array}$ & B. 5 & $\begin{array}{l}15,49 \\
\uparrow 22,22 \text { IT } \\
\sim 8,57 \text { TRA }\end{array}$ & $\begin{array}{l}\mathbf{F}\left(\mathbf{s}^{+}\right): 5,63 \\
8,33 \text { IT; } 2,86 \text { TRA }\end{array}$ & $\begin{array}{l}\operatorname{Pr}\left(\mathrm{s}^{+}\right), \mathrm{IM}, \mathrm{C}, \mathrm{TP},, \varnothing \\
\mathrm{PP}\end{array}$ \\
\hline & $\begin{array}{l}\text { costrutto implicito, simultaneità } \\
\text { nel passato } \\
\text { trainata }(64,79) / \text { trainati }(12,68) \\
(\text { PART })\end{array}$ & A. 2 & $\begin{array}{l}14,08 \\
\uparrow 13,89 \mathrm{IT} \\
\uparrow 14,29 \mathrm{TRA}\end{array}$ & $\begin{array}{l}\varnothing: 7,04 \\
5,56 \text { IT } \\
8,57 \text { TRA }\end{array}$ & $\mathrm{G}, \mathrm{TP}, \mathrm{PP}\left(\mathrm{s}^{+}\right.$?) \\
\hline & $\begin{array}{l}\text { azione extratemporale } \\
\text { significa (Pr) }\end{array}$ & D. 2 & $\begin{array}{l}\mathbf{1 2 , 6 8} \\
\sim 16,67 \text { IT } \\
\sim 8,57 \text { TRA }\end{array}$ & $\begin{array}{l}\text { IM: } 8,45 \\
\text { 11,11 IT; 5,72 TRA }\end{array}$ & $\operatorname{Pr}^{\prime}, \mathrm{C}\left(\mathrm{s}^{+}\right)$ \\
\hline & $\begin{array}{l}\text { simultaneità nella posteriorità nel } \\
\text { passato } \\
\text { sorrideva (IM) } \\
\uparrow\end{array}$ & 2GFC. 2 & $\begin{array}{l}11,43 \\
\sim 5,71 \text { IT } \\
\sim 17,14 \text { TRA }\end{array}$ & $\begin{array}{l}\operatorname{Pr}\left(\mathbf{s}^{+}\right): 7,14 \\
5,71 \text { IT; } 17,14 \text { TRA }\end{array}$ & PR, IM' \\
\hline & $\begin{array}{l}\text { azione centrale nel passato, } \\
\text { visione globale } \\
\text { incominciò }(\mathrm{PR})\end{array}$ & C. 1 & $\begin{array}{l}11,27 \\
\sim 11,11 \text { IT } \\
\sim 11,43 \text { TRA }\end{array}$ & $\begin{array}{l}\text { PP (sn+): } 5,63 \\
8,33 \text { IT; } 2,86 \text { TRA } \\
\text { TP: 5,63 } \\
\text { 2,78 IT; } 8,57 \text { TRA }\end{array}$ & $\begin{array}{l}\text { assenza di soluzioni } \\
\text { inappropriate meno fre- } \\
\text { quenti }\end{array}$ \\
\hline \multirow{6}{*}{$0-10$} & $\begin{array}{l}\text { azione extratemporale } \\
\text { significa (Pr) } \\
\uparrow\end{array}$ & 2D.2 & \begin{tabular}{|l|}
8,45 \\
$\sim 11,11$ IT \\
$\sim 5,71$ TRA \\
\end{tabular} & $\begin{array}{l}\text { IM: } 4,27 \\
\text { 5,56 IT; } 2,86 \text { TRA }\end{array}$ & Pr', C (s+?) \\
\hline & $\begin{array}{l}\text { simultaneità nel passato, visione } \\
\text { corsiva } \\
\text { indossava (PART) }\end{array}$ & GFC. 4 & $\begin{array}{l}7,14 \\
\uparrow 8,57 \text { IT } \\
\downarrow 5,71 \text { TRA }\end{array}$ & $\begin{array}{l}\text { assenza di una } \\
\text { soluzione prevalente }\end{array}$ & $\mathrm{PP}, \mathrm{G}, \mathrm{PR}$ \\
\hline & $\begin{array}{l}\text { simultaneità nel passato, visione } \\
\text { corsiva } \\
\text { indossava (PART) } \uparrow\end{array}$ & 2GFC. 4 & $\begin{array}{l}4,29 \\
\uparrow 2,86 \mathrm{IT} \\
\downarrow 5,71 \mathrm{TRA}\end{array}$ & $\begin{array}{l}\text { assenza di una } \\
\text { soluzione prevalente }\end{array}$ & $\mathrm{IM}(\mathrm{e}), \mathrm{PR}$ \\
\hline & $\begin{array}{l}\text { simultaneità nel passato, visione } \\
\text { corsiva } \\
\text { stava leggendo/leggeva (IM) }\end{array}$ & GFC.6 & $\begin{array}{l}4,29 \\
\uparrow 5,71 \mathrm{IT} \\
\downarrow 2,86 \mathrm{TRA}\end{array}$ & $\begin{array}{l}\text { assenza di una } \\
\text { soluzione prevalente }\end{array}$ & PP, PR \\
\hline & $\begin{array}{l}\text { costrutto implicito, anteriorità nel } \\
\text { passato } \\
\text { massacrati (PART) }\end{array}$ & C. 9 & $\begin{array}{l}4,23 \\
\sim 2,78 \text { IT } \\
\uparrow 5,71 \text { TRA }\end{array}$ & $\begin{array}{l}\text { assenza di una } \\
\text { soluzione prevalente }\end{array}$ & $\begin{array}{l}\mathrm{PR}\left(\mathrm{st}^{+}\right), \varnothing, \text { forma } \\
\text { inesistente }\end{array}$ \\
\hline & $\begin{array}{l}\text { simultaneità nel passato, visione } \\
\text { corsiva } \\
\text { stava leggendo/leggeva (IM) } \uparrow\end{array}$ & 2GFC. 6 & $\begin{array}{l}1,43 \\
\uparrow 2,86 \text { IT } \\
=0 \text { TRA }\end{array}$ & $\begin{array}{l}\text { PP: } 1,43 \\
\text { 2,86 IT; } \\
\text { 0 TRA }\end{array}$ & $\begin{array}{l}\text { assenza di soluzioni } \\
\text { inappropriate meno } \\
\text { frequenti }\end{array}$ \\
\hline
\end{tabular}

Tabella 5: Classificazione dei fenomeni in base alla quantità di risposte inappropriate 


\section{Riassunto}

\section{COMMENTO DELLE SCELTE DELLE FORME VERBALI NEI CLOZE DEGLI STUDENTI DI ITALIANISTICA E DI TRADUZIONE (IDENTIFICAZIONE DEI PUNTI CRITICI E CONFRONTO TRA I GRUPPI)}

Nel presente contributo l'autrice si concentra sull'osservazione e sul commento delle scelte delle forme verbali nei cloze degli studenti del $2^{\circ}$, del $3^{\circ}$ e del $4^{\circ}$ anno del corso di laurea in Lingua e letteratura italiana, del $2^{\circ}$ e del $3^{\circ}$ anno del corso di Laurea in Mediazione interlinguistica e del $4^{\circ}$ anno del corso di laurea in Traduzione. Il punto in comune di tutti e tre i corsi è l'insegnamento esplicito delle caratteristiche e del funzionamento del sistema verbale italiano, anche se nell'ambito di Italianistica la quantità di ore dedicate a questo tipo di insegnamento è superiore rispetto a quella prevista nell'ambito di Traduzione, mentre nell'ambito di Traduzione una quantità maggiore di lezioni concerne esercitazioni di traduzione e composizione di testi. Per gli scopi della ricerca è stato elaborato un test composto da cinque testi autentici brevi ma completi e da un brano tratto dal romanzo Il giardino dei Finzi-Contini di Giorgio Bassani, trasformati in seguito nei cloze. Ma visto che la scelta della forma verbale nei cloze dipende non soltanto dalla conoscenza degli usi, bensì anche dall'interpretazione felice del mondo testuale, i cloze sono stati completati da attività quali sottolineare espressioni sconosciute, tradurre il testo in sloveno, completare i cloze preceduti dalla versione slovena del testo. Dai risultati traspare che gli studenti non hanno problemi particolari nella comprensione del mondo testuale, mentre incontrano difficoltà nell'interpretazione attiva delle sue caratteristiche e nell'applicazione degli usi alla realtà extralinguistica. Per la quantità di usi inappropriati si rivelano come i più critici fenomeni quali l'anteriorità nell'ambito delle frasi indipendenti e relative, l'espressione della temporalità relativa nell'ambito dei costrutti sintattici complessi e nel discorso indiretto libero, il preludio, la scelta tra il perfetto e l'imperfetto e l'espressione di notizie riferite su azioni passate con il condizionale composto. Si è scoperto, però, che la criticità di uno stesso fenomeno varia in dipendenza dalla sua riconoscibilità nel co- e contesto. I risultati hanno inoltre confermato - eccetto che per un gruppo - il miglioramento della prestazione con il progredire degli studi. Al tempo stesso si è potuta notare l'importanza dell'insegnamento esplicito e dell'osservazione attiva degli usi delle forme verbali, dato che né le preconoscenze, acquisite spesso in modo informale e utili soprattutto nella ricostruzione del mondo testuale, né l'esperienza relativa alla composizione di testi possono eliminare l'influsso dell'interferenza. Data la presenza implicita della madrelingua nella produzione linguistica del discente in un'altra lingua straniera, l'autrice propone delle attività in classe che prendano come spunto i testi in madrelingua. Per superare le difficoltà di applicazione delle nozioni teoriche ai casi pratici gli studenti dovrebbero essere esposti ancora di più alla lettura e all'analisi dei vari tipi testo in lingua straniera. Gli studenti dovrebbero essere inoltre incitati a un processo consapevole di ricostruzione del mondo testuale. 


\section{Povzetek}

\section{KOMENTAR K IZBIRI GLAGOLSKIH OBLIK V CLOZIH ŠTUDENTOV ITALIJANISTIKE IN PREVAJANJA (OPREDELITEV KRITIČNIH TOČK IN PRIMERJAVA POSAMEZNIH SKUPIN)}

Avtorica opazuje in komentira izbiro italijanskih glagolskih oblik v clozih študentov 2., 3., in 4., letnika študijskega programa Italijanski jezik in književnost, 2. in 3. letnika Medjezikovnega posredovanja ter 4. letnika Prevajalstva. Skupni imenovalec vseh treh študijskih smeri je prisotnost eksplicitnega poučevanja značilnosti italijanskega glagolskega sistema in njegovih rab, čeprav je delež tovrstnega pouka na italijanistiki večji, medtem ko je na prevajalstvu več aktivnosti tvorjenja besedil. Za namene raziskave je bil izdelan test, sestavljen iz petih kratkih, vsebinsko zaokroženih avtentičnih besedil ter odlomka iz romana Giorgia Bassanija Il giardino dei Finzi-Contini, spremenjenih v cloze. Ker pa pri tem tipu naloge pravilna izbira ni rezultat zgolj poznavanja rabe, ampak najprej pravilnega razumevanja besedilnega sveta, so cloze spremljale dejavnosti, kot so podčrtovanje neznanih besed, prevod besedila v slovenščino in dopolnjevanje besedila ob predhodno podani slovenski različici. Rezultati raziskave pokažejo, da študenti skoraj nimajo težav z razumevanjem besedilnega sveta, pač pa z njegovo aktivno interpretacijo in aplikacijo rab glagolskih oblik. Za najbolj kritične se izkažejo signalizacija preddobnosti v preteklosti v neodvisnih in relativnih stavkih, signalizacija relativnih časovnih odnosov v zapletenejših skladenjskih konstrukcijah in polpremem govoru, signalizacija preludija, izbira med perfektom in imperfektom, signaliziranje ograjevanja pripovedovalca od novic o preteklih dogodkih z obliko condizionale composto. Vendar pa na kritičnost točke ne vpliva zgolj sam pojav, ampak njegova (ne)prepoznavnost v ko- in kontekstu. Rezultati so pokazali - z izjemo ene skupine -, da je napredek premosorazmeren $z$ leti študija. Hkrati se je izkazalo, da je eksplicitno poučevanje in aktivno opazovanje rab glagolskih oblik za njihovo pravilno rabo bistveno, saj tudi predznanje, pridobljeno v neformalnem stiku z jezikom, ki prispeva zlasti $\mathrm{k}$ razumevanju besedilnega sveta, in izkušnje s tvorjenjem besedil ne morejo zajeziti vpliva interference. Zaradi implicitne prisotnosti materinščine pri rabi tujega jezika avtorica predlaga aktivnosti pri tujejezičnem pouku, ki bi izhajale iz besedil v maternem jeziku. Poleg tega meni, da bi študenti potrebovali še več stika s tujejezičnimi besedili, saj bi le tako premostili težave pri aplikaciji svojih teoretičnih znanj v praktičnih situacijah. Prav tako je treba študente spodbujati k zavestni izgradnji in interpretaciji besedilnega sveta. 